\title{
The Clinical Information Systems Response to the COVID-19 Pandemic
}

\author{
J. Jeffery Reeves ${ }^{1}$, Natalie M. Pageler², Elizabeth C. Wick ${ }^{3}$, Genevieve B. Melton ${ }^{4}$, Yu-Heng \\ Gamaliel Tan ${ }^{5}$, Brian J. Clay ${ }^{6}$, Christopher A. Longhurst ${ }^{6}$ \\ 1 Department of Surgery, University of California, San Diego, La Jolla, California, USA \\ 2 Department of Pediatrics, Division of Critical Care Medicine, Stanford University School of Medicine, \\ Palo Alto, CA, USA \\ 3 Department of Surgery, University of California, San Francisco, San Francisco, California, USA \\ 4 Department of Surgery and Institute for Health Informatics, University of Minnesota, Minneapolis, \\ Minnesota, USA \\ 5 Department of Orthopedics, Chief Medical Information Officer, Ng Teng Fong General Hospital, \\ National University Health System, Singapore \\ 6 Department of Medicine, Division of Biomedical Informatics, University of California, San Diego, \\ La Jolla, CA, USA
}

\section{Summary}

Objective: The year 2020 was predominated by the coronavirus disease 2019 (COVID-19) pandemic. The objective of this article is to review the areas in which clinical information systems (CIS) can be and have been utilized to support and enhance the response of healthcare systems to pandemics, focusing on COVID-19.

Methods: PubMed/MEDLINE, Google Scholar, the tables of contents of major informatics journals, and the bibliographies of articles were searched for studies pertaining to CIS, pandemics, and COVID-19 through October 2020. The most informative and detailed studies were highlighted, while many others were referenced.

Results: CIS were heavily relied upon by health systems and governmental agencies worldwide in response to COVID-19.

Technology-based screening tools were developed to assist rapid case identification and appropriate triaging. Clinical care was supported by utilizing the electronic health record (EHR) to onboard frontline providers to new protocols, offer clinical decision support, and improve systems for diagnostic testing. Telehealth became the most rapidly adopted medical trend in recent history and an essential strategy for allowing safe and effective access to medical care. Artificial intelligence and machine learning algorithms were developed to enhance screening, diagnostic imaging, and predictive analytics - though evidence of improved outcomes remains limited. Geographic information systems and big data enabled real-time dashboards vital for epidemic monitoring, hospital preparedness strategies, and health policy decision making. Digital contact tracing systems were implemented to assist a labor-intensive task with the aim of curbing transmission. Large scale data sharing, effective health information exchange, and interopera- bility of EHRs remain challenges for the informatics community with immense clinical and academic potential. CIS must be used in combination with engaged stakeholders and operational change management in order to meaningfully improve patient outcomes.

Conclusion: Managing a pandemic requires widespread, timely, and effective distribution of reliable information. In the past year, CIS and informaticists made prominent and influential contributions in the global response to the COVID-19 pandemic.

Keywords

Coronavirus, pandemic, electronic health record, clinical

information systems, telehealth

Yearb Med Inform 2021:105-25

http://dx.doi.org/10.1055/s-0041-1726513

\section{Introduction}

The magnitude of the coronavirus disease 2019 (COVID-19) pandemic has permeated every area of healthcare, academia, politics, and industry such that this survey must begin with a brief history. Initially identified in December 2019 following an outbreak of atypical pneumonia in Wuhan, China, COVID-19 was declared a global pandemic by the World Health Organization (WHO) on March 11 $1^{\text {th }}, 2020$
$[1,2]$. Through November 2020, more than 62 million cases of COVID-19 have been reported in 191 countries leading to more than $1,400,000$ deaths [3]. The ensuing toll on the world's population is impossible to quantify. The global response has included exhaustive case identification efforts through screening and diagnostics, governmental responses and regulations to limit transmission, comprehensive preparations for expanding hospital capacity, and an unprecedented search for novel therapeutics and effective vaccination [4-6] The scientific community responded with a historic influx of research, with now over 280,000 scholarly articles pertaining to COVID-19 [7]. We are proud to report that clinical informaticists and the field of biomedical informatics are playing a prominent role in addressing COVID-19 [8]. Pandemic management occurred at multiple levels via international, national, regional, and local public and private institutions [9-11]. Though varied in detail, standard approach- 
es emerged, and a universal theme arose the need for timely and reliable information to enable critical decision making [12, 13]. The novelty of the crisis and speed of change required continuous communication and distribution of data and knowledge to allow development, dissemination, and adoption of evidence-based practices [13-15]. Digital technologies have been employed globally to support and enable public-health responses to COVID-19 at an unprecedented scale [16].

Recent surveys in the IMIA Yearbook of Medical Informatics define Clinical Information Systems (CIS) as a "set of resources, techniques, devices, and methodologies used to support the needs of healthcare organizations" whose crucial role is "to capture, store, process, and transfer information to clinical decision makers" [17, 18]. By integrating vital information in the daily practices of healthcare administrators and front-line providers, CIS can serve as a public health tool during acute crises [19]. The subsequent collection and storage of patient data and provider actions creates an invaluable source of data with myriad applications. Given the rapid rise of telehealth as a safe and effective mode of healthcare delivery in the era of physical distancing, it was an influential year for CIS $[16,20,21]$. An electronic health record (EHR) system is now utilized by $>95 \%$ of hospitals in the United States of America (USA) and $>80 \%$ of office-based practices in both the USA and the European Union [22, 23]. Globally, $47 \%$ of countries have a national EHR and $70 \%$ of WHO member countries have an eHealth policy [24]. The prevalence of EHRs necessitates involvement of technology to facilitate both small- or largescale clinical management strategies that reduce administrative burdens and enhance patient care [19]. The aim of this survey is to summarize pertinent articles specific to COVID-19 and the areas in which CIS have been applied during the pandemic to improve patient care. Given the volume and rapidly evolving nature of the literature, we do not attempt to be comprehensive. Rather, we highlight some interesting areas of research and operations to discuss opportunities and future directions.

\section{Methods}

We conducted a review of the literature limited from the year 2019 through the end of October 2020 utilizing PubMed/MEDLINE and Google Scholar databases by combining the search terms "clinical/health information systems", "health/medical/ clinical informatics", "health information technology", "digital health", "electronic health/medical record", "and health information exchange" with "coronavirus", "COVID-19", "severe acute respiratory syndrome 2 (SARS-CoV-2)", "pandemic", "pandemic/outbreak management". We then searched the table of contents of major health informatics journals such as the Journal of the American Medical Informatics Association (JAMIA), the International Journal of Medical Informatics (IJMI), and Applied Clinical Informatics (ACI) for relevant original reports. Finally, we reviewed the reference section within each selected article to identify additional potentially relevant articles. The articles selected for detailed review are listed in Table 1.

\section{Clinical Information Systems and Antecedent Infectious Disease Outbreaks}

Prior to the emergence of the severe acute respiratory syndrome coronavirus 2 (SARS-CoV-2), several other epidemics and pandemics have occurred during the digital era, representing opportunities to use CIS in outbreak management. In 2003, following the outbreak of the first severe acute respiratory syndrome (SARS) in China, a web-based integrated database (SARSID) was used to collect hospital-based clinical data from SARS patient wards [25]. Similarly, global information systems such as the WHO's Global Outbreak Alert and Response Network were used to facilitate rapid sharing of new information pertaining to SARS [26, 27]. Zhao et al. in 2010 described an attempt by the Chinese government to construct regional and national health systems utilizing information and computer technology to prepare for any subsequent outbreaks of emerging infectious diseases (EID) like SARS [28]. Public health authorities in the case of SARS recognized the ability of electronic systems to integrate health resources, collect patient data, and share medical information across regions in order to benefit public health [28]. Chen et al. published an elegant study in 2011 discussing the various information systems-related public health initiatives that were undertaken as a result of SARS [29]. These included national electronic disease surveillance systems, health information exchanges, and electronic health record alerting; all of which were intended to assist the management of EID [29]. In addition, the authors analyzed news coverage and contact tracing data from SARS patients in Taiwan to develop an alternative framework for outbreak management. They argued that most public health strategies typically emphasized strengthening central control and management. However, EID are often clinically ambiguous and effective control requires local ability to detect unusual cases. Public health information systems should therefore promote and facilitate local discussion, investigation, and recognition of important outliers and timely dissemination of information to improve detection of novel diseases. Such systems can be supported by well-designed CIS. Mandl et al. in 2004 described the opportunity to develop syndromic surveillance systems to detect discernable clinical case features of a given disease before confirming the diagnosis [30]. Initially intentioned for detection of a bioterrorist attack, the authors point out that such a system would also be useful for public health and detection of infectious diseases. Six years after SARS, the USA encountered the highly contagious strain of the influenza A virus, subtype H1N1 [31]. In June 2009 a pandemic was declared, and between 150,000 and 575,000 people died from the virus worldwide [31]. In response, a similar call was made for the design of a multinational informatics infrastructure with standardized data and indicators for information collection and disease surveillance [32]. In 2011, a group from Columbia University configured a widget-based system to provide clinical decision support (CDS) with automatic 
retrieval, alerts, and advice for primary care providers seeing patients with suspected H1N1 infection [33]. In 2014, Keck et al. published an interesting study in the JAMIA about using the EHR for influenza surveillance in the American Indian and Alaska Native (AI/AN) populations [34]. In the USA, the Indian Health Services (IHS) utilizes a nationalized EHR-based health information technology platform and provides healthcare to 2.56 million $\mathrm{AI} / \mathrm{AN}$ citizens in multiple geographic locations across 37 states, including remote villages [35]. Within four weeks of the first recognized 2009 H1N1 cases in the USA, an EHR-based surveillance system, the IHS Influenza Awareness System, was created and implemented using algorithms based on International Classification of Diseases, Version 9 (ICD-9) codes, Current Procedural Terminology (CPT) codes, and other routinely collected clinical data. The software searched 162 databases across 343 health facilities and detected influenza-like illness visits with a sensitivity of $96.4 \%$ and a specificity of $97.8 \%$. This system facilitated the timely and accurate detection of influenza-like illnesses and illustrated the potential capabilities of EHR-based public health surveillance. In March of 2014, an outbreak of Ebola Virus Disease emerged in West Africa and eventually led to more than 28,600 cases and 11,325 deaths over the course of two years [36]. Following this more recent epidemic, several authors highlighted the opportunity to utilize CIS to support local and public health management. Landman et al. described the use of CDS in their local hospital to require Ebola screening upon presentation to the emergency department [37]. Oza et al. built an interoperable EHR that included a tablet-based application designed to address the challenge of data collection in a highly infectious environment [38]. Mobile phone data was used to track the patterns of human mobility across West Africa, estimate the spread of disease, and was featured in a feasibility study of an electronic system for contact tracing [39, 40]. The US Department of Defense created an Electronic Surveillance System for the Early Notification of Community-based Epidemics (ESSENCE) which screens the "reason for visit" in the
EHR [41]. ESSENCE monitors the Military Health Systems EHR, provides alerts for potential EID, and allows epidemiologists and public health officers to investigate reportable disease events. The US Department of Commerce published a report on EHR design considerations in responding to highly infectious diseases, offering 18 measures to augment existing EHR functionality to increase public safety [42]. In the 2015 IMIA Yearbook, Borycki et al. provided a review of the literature describing how technology and EHRs can be used to support safe and patient-centered care regarding Ebola [43]. They outline the ability to use the internet, mobile applications and social media, EHRs, and CDS to support patient-centric care in times of emerging diseases and epidemics. They also detail the EHR design, human factors issues, policy and organizational issues, reporting, and quality improvement efforts utilizing health information technology (IT). Finally, Mandl wrote a viewpoint in the JAMIA about the potential to use the EHR as a public health tool across the USA in response to Ebola [44]. He highlighted many of the points described above and offered simple interventions to mitigate the socio-administrative and regulatory barriers that slow progress in health IT. Overall, past experience with EID demonstrated the potential to develop CIS that can surveil populations, rapidly identify new cases, offer immediate screening and CDS in order to limit transmission, gather large amounts of data, and enable broad communication. Unfortunately, huge populations, geographical variance, and disparity of health resources create significant barriers to widespread implementation of such systems. The global health informatics infrastructure remains highly fragmented. As of 2015, more than a decade following the outbreak of SARS, only $57.5 \%$ of hospitals in China participated in the regional medical consortium designed to enhance health information exchange (HIE) [45]. Even within countries with highly digitalized healthcare such as the USA, the infrastructure needed for effective HIE varies dramatically [46]. The full realization of CIS to mitigate the spread of infectious diseases was therefore not present when COVID-19 first emerged in Wuhan.

\section{Clinical Informatics to Support the COVID-19 Response within Health Systems}

Reeves et al. in JAMIA were the first group to publish a description of healthcare informatics supporting a rapid COVID-19 response at a health system [19]. Clinicians from the University of California, San Diego (USA) treated some of the earliest cases in the USA, including patients evacuated from Wuhan, China on February 7 , 2020 [47]. Anticipating further infections, Reeves et al. detail the rapid configuration of EHR-based tools to support outbreak management. First, they report multi-modal electronic screening and triage processes to limit exposures to on-site personnel and patients. Second, standardized EHR-based note templates were created and used across multiple settings to help front-line care providers support hospital protocols by offering up-to-date information at the point of care. Third, CIS helped with appropriate testing, isolation, and ancillary orders. Fourth, an existing telehealth infrastructure was rapidly expanded, and providers were trained to transition away from in-person care when appropriate. Finally, EHR-based real time reports and an operational COVID-19 dashboard were created for use by the local incident command center in order to guide institutional decisions. A second early report written by Grange et al. in ACI detailed the rollout of IT services support for the clinical response at the University of Washington, one of the first endemic hotspots in the USA [48]. To enable rapid diagnostic testing, an in-house laboratory test was validated, unambiguously named, and embedded in a standardized set of orders that was made available to physicians and triage nurses. Their laboratory information systems were configured to interface with the EHR and external systems for public health reporting. CDS was used to provide direct access to informational hyperlinks, ICD, $10^{\text {th }}$ Revision (ICD-10) diagnoses, billing codes, and discharge instructions. Finally, telehealth was utilized in the intensive care setting to allow practitioners to perform bedside visits while 
conserving personal protective equipment.

Lin et al. in JAMIA described an interesting use of informatics at the University of Colorado (USA) to assist with the rapid onboarding of staff and physicians [49]. There was a need to educate providers with no recent experience in hospital medicine who were recruited to help manage a potential surge. A comprehensive training guide with a series of videos and resources was not only posted online, but it was also embedded into the EHR such that it could be accessed any time at the point of care. They also described efforts to improve communication with hospitalized patients' family members not physically present at the bedside through use of tablets. Finally, nurses unable to work on the wards utilized secure messaging within the EHR to communicate with bedside nurses, physical therapists, chaplains, and social workers. This re-purposed workforce gathered information about hospitalized COVID-19 patients and relayed updates to family members. Milenkovic et al. in IJMI describe the adaptation of CIS at the University of Nis and the largest healthcare institution in the Republic of Serbia [50]. First, they created a patient triaging module utilizing deep neural networking based on questionnaires. They also developed an algorithmic scheduling model to group patients into high-risk categories that automatically scheduled into appropriate time slots. Prescriptions of chronic medications were extended to decrease the need for in-person visits. In addition, new reporting modules were created, and short message service (SMS), laboratory, and mail notification systems were expanded. Overall healthcare volume declined as the authors report effective social distancing was enabled through use of these various CIS modules. Yan et al. analyzed 368 webpages across 50 hospitals in mainland China and identified the following five focal themes of how IT was used to respond to the pandemic [51]: (1) popular medical science education; (2) digitalized hospital processes; (3) knowledge management for medical professionals; (4) telemedicine; and (5) new IT initiatives. Ye et al. in the Journal of Medical Internet Research (JMIR) Medical Informatics describe the framework of IT more broadly used to manage the pandemic throughout the country of China [52].
In this framework, government agencies, technology companies, medical facilities, and research institutions used technology including internet-based services, big data, predictive analytics, cloud computing, and AI for information delivery, screening, risk assessment, tracking, intelligent diagnosis, and telemedicine. Applied technology enabled detection, early response, intervention, and post-intervention processes to improve appropriate isolation and care. A common conclusion is that the EHR is an essential tool to support the clinical needs of a health system during a pandemic. However, EHR systems are not universally adopted. Faced with a different challenge, clinical informaticists at the Martinique University Hospital in France responded to the pandemic where they practiced without an EHR [53]. Sylvestre et al. describe in the JAMIA how after initially relying on classic outbreak monitoring with manual reporting, they developed simple technology-based tools to respond to rapidly evolving situations. Databases for triaged outpatient potential cases and hospitalized patients were built, and a web-application to enable monitoring, perform queries, and create real-time reports was created. Given globalization and large populations, technology and CIS were able to add significant benefit to otherwise manual processes. Kannampallil et al. from Washington University in St. Louis (USA) present their experience as informaticists engaging in an operational role during the pandemic and share four critical lessons applicable to all [54]: (1) work together, mitigate barriers, and fill data management gaps; (2) develop locally, share regionally and nationally; (3) adapt rather than build, and deliver knowledge at the right time, place, and format; and (4) support an evolving research enterprise.

Each of these authors report several challenges in completing extensive informatics build in a short period of time. One vital strategy also reported was the distinct benefit and need to include informaticists in the institutional incident command center structure to ensure proper prioritization and design of solutions to address health system needs. Many of the EHR-based tools mentioned above enable rapid process adaptation and can improve patient care. However, we found no objective evidence to show a benefit in medical outcomes with the use of these EHR-based tools. The literature in this area is largely descriptive due to the impracticality of well-controlled prospective or retrospective studies when rapidly responding to a global pandemic. Regardless, in the age of the EHR, it is clear that leveraging CIS and healthcare informatics expertise are important to a successful response and integral to the health system's response in addressing COVID-19. In addition to the EHR, one of the most important aspects of CIS throughout the pandemic has been the rapid and near universal expansion of telehealth services.

\section{Telehealth}

Over the last two decades, many healthcare systems have been slowly rolling out telehealth services. According to 2017 and 2018 American Hospital Association Survey data, more than $60 \%$ of hospitals reported adoption of some form of telehealth and more than $13 \%$ had tele-intensive care unit capabilities prior to the COVID-19 pandemic [55]. Prior to COVID-19, there was wide variation in deployment of telehealth infrastructure and utilization across the world and within individual countries, for example, in the USA there was slightly more utilization in rural areas [56, 57]. Historically, the most commonly cited barriers to telehealth included poor reimbursement, lack of provider interest, regulatory barriers, lack of time or resources for training, insufficient telecommunications technology and infrastructure, privacy concerns, and patient preference for in-person care [55, 58-60]. The COVID-19 pandemic provided sufficient incentives to simultaneously overcome many of these barriers in a very short time period. Given the concurrent needs to facilitate social distancing, minimize unnecessary infection exposures, and preserve personal protective equipment (PPE), patients and providers quickly reached to telehealth and related digital health solutions. As the number of COVID-19 patients increased in the early months of 2020, there was a tightly correlated spike in internet search volume for telehealth and telemedicine in the USA $[55,61]$. Partially based on previous experience with 
global emergencies, health care professionals quickly recognized the potential for telehealth to meet emerging healthcare needs during the pandemic [20,21, 62]. Mehrotra et al. published one of the first accounts of rapid transition to telehealth within weeks of the public health emergency in four physician practices across the USA [63]. Of note, they highlighted heavy dependence on telephone over video visits because of technical, training, and workflow challenges. In contrast, as noted above, Reeves et al. also published one of the early reports of telehealth use in the pandemic as part of a broader clinical informatics response and highlighted the role of both existing telehealth infrastructure and redeployed clinical informatics team members to facilitate extremely rapid training and expansion of telehealth services [19, 64]. Mann et al. published in JAMIA their experience at New York University, an early epicenter of the USA COVID-19 outbreak [65]. They noted initial greater than 2-fold growth of urgent care telehealth visits, quickly followed by greater than 40 -fold growth in ambulatory visits, with consistently high patient satisfaction scores. As with the University of California, San Diego (UCSD, USA) group, these visits were primarily video-enabled on pre-existing telehealth infrastructure. In response to the pandemic, there was a rapid growth in applications and adoption of and use cases for telehealth in the ambulatory setting in almost every specialty from mental health to oncology to obstetrics [66-70]. There was also widespread extensions of telehealth utilization beyond evaluation and management by independent practitioners to provisions of a wide range of virtual therapy from physical therapy to occupational therapy to psychotherapy $[66,71,72]$. This extended spectrum of ambulatory telehealth use cases was partially enabled by the changes in legal and reimbursement policies discussed below. While telehealth adoption occurred first in the ambulatory setting following the COVID-19 outbreak, there quickly followed a spike in telehealth utilization in the inpatient setting as well [73]. Especially as shortages of PPE emerged, hospitals turned to novel applications of telehealth to minimize PPE requirements, enable social distancing, and minimize potential exposure to patients, families and staff. Inpatient telehealth applications included virtual multi-disciplinary rounds, telehealth consults both to isolated patients and from remotely located providers, communication/ education with family members who were remote due to restricted hospital visitation policies, visual monitoring of patients in isolation, and virtually assisted patient resuscitation, amongst other novel applications [74-76]. Vilendrer et al. convened three very different health systems - an academic medical center, a teaching hospital, and a safety net county hospital - to share lessons learned [75]. The differences in patient demographics and available technology drove different use cases and design of solutions. However, all three organizations relied on established relationships with their vendor partners, pre-existing video conferencing solutions, and readily available consumer-grade hardware for rapid deployment. All three institutions identified ongoing gaps in translation services, hardware maintenance, and optimized security protocols. These solutions and implementation challenges are consistent with other case reports of rapid inpatient telehealth implementations [74, 76]. While most of the published reports to date describe only process metrics and lessons learned from rapid implementation of inpatient telehealth, Jones et al. evaluated glycemic levels in patients before and after implantation of a virtual inpatient diabetes co-management service and found no clinically significant differences [77]. In addition to the pure clinical applications, there was early recognition that telehealth could be used to maintain associated clinical activities such as medical education and clinical research during pandemic conditions [78]. Direct observation and participation in clinical care is a critical component of medical education, yet in response to the need to minimize infection exposure and enable social distancing, many medical students and other trainees were initially excluded from the clinical setting or thrust into new modalities of care that disrupted traditional clinical supervision practices. Creative deployment of telehealth technologies such as virtual rounds [79] and proctored telehealth visits [80] enabled trainees to safely rejoin clinical conversations and participate in clinical care
[81]. Similarly, where appropriate, telehealth has also been recommended and deployed to enable the continued conduct of clinical trials while minimizing risks to participants [82]. There are several factors which have contributed to the successful, rapid, widespread deployment of telehealth in the response to the public health emergency. Prior to the COVID-19 pandemic, regulatory issues and poor reimbursement had been repeatedly cited as barriers to deployment of telehealth services worldwide [59, 60, 83]. A strong incentive to focus resources on keeping patients and staff safe spurred governmental interventions to remove or decrease these barriers. In the USA, the Centers for Medicare and Medicaid Services rapidly expanded reimbursement for a broad range of telehealth services to beneficiaries and federal agencies encouraged and incentivized providers to implement telehealth services [84-86]. Importantly, these provisions extended to clinicians who may not independently bill for evaluation and management, including rehabilitation therapists and clinical psychologists [84]. Another critical policy change in the USA allowed temporary waivers for state-specific licensure [87]. Finally, enforcement of the Health Insurance Portability and Accountability Act was relaxed to allow broader access to communications technologies for providers and patients [88]. Similar regulatory relaxations and additional telehealth incentives were adopted in various countries and provinces around the world $[60,83]$. Most of the literature on telehealth deployment during the COVID-19 pandemic has focused on the potential and realized benefits, but there are important unintended consequences that deserve specific attention. While telehealth can improve healthcare access and ease the burdens of transportation, child care, time off work, etc. for families with limited resources, there has been growing recognition of the potential for telehealth to exacerbate disparities because of variable access to internet services and technology and varying levels of digital literacy [89]. Even as telehealth has been broadly deployed during the pandemic to reduce infectious exposure and reach patients whose travel was limited, there has been quick recognition of particularly vulnerable populations who are being disen- 
franchised by the evolving modes of healthcare delivery $[90,91]$. Nouri et al. evaluated the utilization of healthcare services in the University of California San Francisco primary care practices before and after widescale telehealth implementation at the beginning of the COVID-19 pandemic and showed statistically significant decreases in healthcare utilization by patients older than 65 years old, non-English speaking patients, and Medicare and Medicaid patients compared to their peers [91]. They outline a series of interventions to address barriers to telehealth adoption in vulnerable populations including interventions to increase digital literacy, provision of mobile devices, educating about free/low-cost broadband internet access, integration of interpreters, actively screening for barriers when scheduling visits, and advocating for policy and infrastructure development. As with all areas of health informatics innovation and research, clinical informaticists have a responsibility to critically assess and address inequities that are created or exacerbated as new telehealth technologies are deployed [92]. The practice of medicine has been irrevocably transformed by the surge of telehealth deployment during the COVID-19 pandemic. While it is certain that we will not revert completely to the pre-pandemic traditional practice of medicine, the precise landscape of post-pandemic telehealth remains to be seen. The recent investment in telehealth has helped establish long-lasting technical infrastructure to support telehealth going forward, but the policies that reduced telehealth barriers may easily be reversed. A wide range of permanent policy changes, infrastructure development, and outcomes research needs to be done to optimally integrate telemedicine and other digital health technologies into the future practice of medicine $[20,21]$. Furthermore, we need to better understand the effect of telehealth interventions on specific patient populations and to delineate which types of care are most amenable to this care modality. Prior to the pandemic, there were limited rigorous studies on patient outcomes associated with telehealth interventions [93]. The dramatic expansion of the telehealth implementations and use cases only exacerbates the need for rigorous research into the effects of this rapid transition in healthcare delivery. Clinical informaticists, who have a broad understanding of the people, process, and technical issues involved in ongoing telehealth optimization, are perfectly poised to lead the next phase of research and innovation.

\section{Case Identification, Remote Monitoring, and Screening}

Cohesive CIS across traditional healthcare, ambulatory, and community settings can create improved outbreak management and mitigation strategies at scale. As COVID-19 caused near universal travel restrictions, some nations were able to curb transmission by using technology to effectively screen individuals as they crossed borders [94-96]. Wang et al. in JAMA published the first and most robust report of electronically-assisted travel screening in Taiwan, a country of more than 23 million people [14]. A mere seven days after the Taiwan Centers for Disease Control (Taiwan CDC) activated their Central Epidemic Command Center, the National Health Insurance Administration and National Immigration Agency integrated patients' travel history and patient identification cards into a unified database [14]. Government officials were then able to immediately identify those who: (1) were at risk due to recent travel in affected areas; (2) reported concerning symptoms during a clinical visit or online; and (3) were confirmed to be SARS-CoV-2 positive. They also utilized sensor data from mobile phones to identify those who may have been in contact with passengers from cruise ships known to have laboratory-confirmed outbreaks [97]. Furthermore, all hospitals, clinics, and pharmacies in Taiwan were granted access to patients' travel histories to assist provider assessment and identification of additional potential cases. Following identification, individuals were automatically notified of risk status. Those entering the country classified as low risk were sent a health declaration border pass to personal mobile phones for expedient immigration clearance. Those identified as high risk, as well as domestically located patients with concerning symptomatology or other exposure, were notified to quarantine at home. The Taiwan CDC then surveilled and recorded daily health statuses of quarantined persons through the use of an electronic tracing system, termed the Infectious Disease Contact Tracing Platform and Management system [98, 99]. In addition, the Taiwan CDC published a daily report of contact tracing data of individuals who were exposed to imported or indigenous confirmed COVID-19 cases [100]. The Taiwanese government has been heralded for these and other public health measures as a model example for transmission prevention. However, others express concern of violation of privacy rights [101]. As of November $28^{\text {th }}, 2020$, Taiwan has recorded just 648 confirmed cases and 7 deaths due to COVID-19 [3, 102]. For most countries, locally transmitted cases quickly became the predominant force driving new cases, and screening became the responsibility of local or regional public health officials and health systems [2]. Virtual screening systems were used to identify symptomatic patients who needed testing without requiring additional physical exposures [103]. Judson et al. at the University of California, San Francisco (UCSF) in the USA designed and deployed an electronic self-triage and self-scheduling tool to assist with efficient and appropriate dispositioning [104]. Accessed by patients through the EHR-tethered patient portal, the tool provided a series of questions about exposure, symptoms, and comorbidities. Branching logic determined the recommended visit type, allowed for direct scheduling, and provided advice and information. The tool was completed by asymptomatic (28\%) and symptomatic (72\%) patients who were triaged to emergent $(24 \%)$, urgent $(24 \%)$, non-urgent $(12 \%)$, or self-care (40\%). The sensitivity of detecting an emergency-level illness for patients who completed the self-triage tool was $87.5 \%$ (95\% CI 72.0-79.5\%) and the specificity for recommending self-care was $89.5 \%(95 \% \mathrm{CI}$ $84.6-93.2 \%)$. Of the patients triaged to selfcare, $61 \%$ had no further interaction with the healthcare system during the subsequent two days, suggesting the ability to reduce unnecessary encounters. Furthermore, the median time to having a scheduled appointment decreased by over two hours. Overall, the tool demonstrated an ability to improve 
triaging efficiency while decreasing unnecessary emergency department visits, thereby limiting unnecessary exposures. Judson et al. also created a chatbot-based employee screening tool at UCSF to facilitate front-entrance screening of healthcare workers [105]. Managing patients remotely was an important strategy to prevent overwhelming an overburdened system. Facing limitations in testing capacity, Annis et al. from the University of Minnesota (USA) configured and deployed an electronic patient education and a remote patient monitoring program for patients with diagnosed or presumed COVID-19 [106]. Utilizing interactive technology, the platform provided patients with disease information, reminders to physically distance and maintain hygiene, and daily symptom questionnaires to monitor progression. Patients had the ability to directly send messages to the care team and any potentially concerning results from the questionnaires were flagged for manual review. The overall activation rate of the program was $61.2 \%$ and the completion rate was $62 \% .94 \%$ of patients who activated accounts checked in at least once and only $6.6 \%$ ultimately required an emergency department visit or hospital admission. The authors report the greatest benefit based on patient comments was a sense of safety from quick access to COVID-19 specific care. Ford et al. from the Medical University of South Carolina (USA) created several remote patient care tools via a COVID-19 registry in the EHR [103]. This included remote home monitoring, an integrated smartphone app with biosensors, and a dashboard to follow patient risk and disease progression. Perlman et al. describe a digital health application that offers self-assessment, an artificial intelligence-driven symptom checker, and communication with remote physicians if necessary [107]. For in-person encounters, electronic travel and symptom screening protocols embedded directly at the point of care helped to ensure compliance as patients accessed healthcare facilities [19]. Perez-Alba et al. utilized a quick response $(\mathrm{QR})$ code posted on walk-in clinic walls to self-administer a questionnaire and provide information to staff in order to risk stratify, appropriately isolate, and prioritize patients without making direct contact [108]. In an effort to conserve
PPE, Turer et al. from Vanderbilt University (USA) in JAMIA coined the term "electronic PPE" (ePPE) [109]. They define ePPE as the use of "telemedicine tools by on-site emergency providers to evaluate patients physically present in the (emergency department) to avoid physical proximity". The use of ePPE allowed the safe triage of suspected patients. In addition to outlining their workflow with ePPE, the authors discuss legal implications as on-site physicians in the USA were previously required to perform medical screening exams that often involve a hands-on in person physical examination.

\section{Diagnostic Testing}

Accurate and timely diagnosis is key to pandemic management. The gold standard for diagnosis of infection with SARS-CoV-2 is reverse transcription polymerase chain reaction (RT-PCR) testing [110]. Unfortunately, there have been challenges with laboratory-based testing given the limited supply of test kits and a substantial burden on the normal processing of laboratories. With the struggle to increase testing capacity, many researchers have sought to use technology and CIS to help alleviate the situation by: improving screening and triaging to reduce unnecessary testing $[111,112]$; improving risk stratification to prioritize testing [113, 114]; enhancing laboratory information systems (LIS) to reduce processing time [115]; and (4) supporting alternative mechanisms of diagnosis [116]. Weemaes et al. in JAMIA described the design, implementation, and requirements of the LIS necessary to support an exponential influx of SARS-CoV-2 testing at the Belgian National Reference Center for COVID-19 testing [115]. The authors detail how the LIS can help remove common bottlenecks across the following five phases of laboratory-based testing: (1) Pre-laboratory phase: computerized order entry and COVID-19 order sets aid in standardized and uniformed ordering; digital notifications are used to improve sample collection and shipping instructions; (2) Pre-analytical phase: computerized order entry alleviates burden on administrative staff for sample registration; automated scripted triaging enables quick and adaptable testing triaging; sample tracking enables easy retrieval for rapid testing, select sampling for studies, and individualized turnaround time estimates; (3) Analytical phase: bidirectional interfacing alleviates burden on laboratory technicians; (4) Post-analytical phase: statistical flagging of outliers for automated validation; automated fax and encrypted email enables automated reporting alleviating burden on administrative staff and call centers; and (5) Post-laboratory phase: automated email, text messages, sample tracking are used for epidemiological reporting and study; computerized order entry enables index patient tracking; searchable database allows for easily retrievable detailed information on each sample and daily summary statistics to assist in data-driven adjustments in crisis management.

\section{Artificial Intelligence in Diagnosis and Predictive Analytics}

At the onset of the outbreak, statistical modeling was heavily relied upon to forecast disease incidence, hospital censuses, ventilator requirements, and deaths [117-121]. These models were useful for policy makers and hospital administrators preparing for regional surges. As Combi and Pozzi's detail in their recent IMIA Yearbook Survey, the application of artificial intelligence (AI) in healthcare has become a popular area of research [17], and many now assert the potential for AI systems to contribute to containment of the COVID-19 pandemic [122]. Debnath et al. wrote a perspective in Bioelectronic Medicine outlining the points in a typical patient care pathway where machine learning and AI systems can potentially augment clinical decisions [123]. These include diagnosis and screening, initial and ongoing risk stratification, prognosis and prioritization of care, and allocation of resources. Obeid et al. in JAMIA describe an application of natural language processing and AI to improve their virtual care screening algorithms [111]. During their telehealth consultation and screening visits, data is 
directly entered by symptomatic patients via both a template-based form and free text entry. The resulting data was analyzed for differences in word frequencies between patients who ultimately tested positive and negative for COVID-19. Interestingly, words mentioned in higher frequency by patients who tested positive included those such as "smell", "taste", "sense", and "loss". This analysis was embedded in a convolutional neural network (CNN) AI model for predicting COVID-19 positivity based on patients' reported symptoms. The performance of the model was relatively low, with an area under the curve (AUC) of 0.729, limiting its usefulness and generalizability. A more frequent application of AI was in the diagnosis of COVID-19 based on radiology studies. Zhang et al. in Cell detail the development of one such system based on chest computed tomography (CT) images [124]. The authors used a large database with images from patients with novel coronavirus pneumonia $(n=3,777)$, other common forms of nonCOVID-19 pneumonia, and normal controls. Their AI system consisted of two models, a lung-lesion segmentation model and a diagnosis prediction model. They used clinical and radiological features to create a prognostic model to predict progression to critical illness. The AI system was tested and validated in an initial retrospective cohort study, three prospective pilot studies within separate Chinese provinces, and a final open source and international prospective pilot study. The results were promising, demonstrating an overall accuracy of $92.49 \%$ and an AUC of 0.9813 (95\% CI: 0.9691 0.9902), establishing an ability of AI systems to assist in rapid diagnosis and prognosis of COVID-19. Li et al. in Radiology published similar results in utilizing a deep-learning AI model to differentiate novel coronavirus pneumonia from other forms of community-acquired pneumonia based on CT images [125]. Murphy et al. published a study in Radiology of a deep learning-based AI system that analyses frontal chest radiographs rather than CT images [126]. The system was compared with the performance of six independent thoracic radiologists and in most cases was found to perform at the same level or better. Achieving an AUC of 0.81, the authors show the potential to utilize machine learning algorithms to support radiologists in resource-limited settings who may not have access to CT imaging. Both Zhang et al. and Murphy et al. made their models publicly available online. Similarly, Hurt et al. from UCSD published a deep learning localization of pneumonia for COVID-19 assessment in the Journal of Thoracic Imaging [127]. This work was rapidly implemented as a cloud-based tool that processed every frontal chest radiograph and chest CT across the UCSD health system. Carlile et al. subsequently found that $86 \%$ of emergency department physicians agreed that the intervention was easy to use in their workflow, and $20 \%$ of respondents reported that the algorithm impacted clinical decision making [128]. In a different approach, Goodman-Meza et al. in PLOS One described the creation of a machine-learning algorithm that uses blood laboratory values to predict the presence or absence of a COVID-19 infection [129]. Pattern recognition of characteristic changes in the complete blood cell count and elevations in inflammatory markers such as C-reactive protein and lactate dehydrogenase were included from 182 COVID-19 positive patients. The model achieved a sensitivity of $0.93(95 \%$ CI $0.85-0.98)$ and specificity of $0.64(95 \%$ CI $0.58-0.69)$ with an AUC of 0.91 (95\% CI 0.87-0.96). Their results show that ancillary laboratory values and machine learning algorithms may be used as screening tools in settings where PCR testing is limited without the use of chest CT. Mei et al. in Nature Medicine integrated chest $\mathrm{CT}$ findings, clinical symptoms, exposure history, and laboratory values in comparing three diagnostic $\mathrm{AI}$ algorithms [116]. The first was based on chest CT images and two levels of CNNs (abnormal slice selection followed by diagnosis). The second used demographic and clinical data within a machine learning model. Finally, features generated by the first two models were integrated by multilayer perceptron to produce the final output of the algorithm. Among a test set of 279 patients, the AI system had comparable sensitivity with a senior thoracic radiologist. However, one key finding was that of 25 patients with a normal chest CT at presentation, $17(68 \%)$ were classified at COVID-19 positive by the AI model, where- as $0(0 \%)$ were classified as positive by the senior thoracic radiologist. The major application of such a system could be as a useful triage tool for positively identified patients, particularly those with otherwise negative imaging, while awaiting definitive PCR results. Liu et al. created a dynamic risk assessment decision support system that was used by Chinese general practitioners in Zhejiang Province during the outbreak [112]. They constructed a dynamic risk stratification model based on a multiclass logistic regression algorithm to classify patients as low, medium, or high risk of having COVID-19 infection. Patient demographics, clinical symptoms, contact history with or without blood results, and CT imaging were used in the model to produce high sensitivity and specificity. The model was used to assist general practitioners in assessing the appropriate risk, triage, management, and follow-up for suspected patients. Liang et al. in Nature Communications applied a deep-learning neural network-based survival model to predict the risk of critical illness for patients diagnosed with COVID-19 [130]. Impressively, the model, which uses ten clinical variables, was developed from data obtained from 575 medical centers across China. Following internal and external validation, they created an online tool embedding the model within a simple nomogram which predicted the probability of critical illness within 5 , 10 , and 30 days. This model offers a user-friendly way to use AI to triage patients at the time of hospital admission. A common limitation to all of the aforementioned studies was the small volume of COVID-19 positive patients that were used as data points. In an effort to support research and development of AI technology, Jacob et al. developed a National COVID-19 Chest Imaging Database in the United Kingdom (UK) [131]. Briefly described in the European Respiratory Journal, the authors highlight the long-standing challenge of data-sharing and acquisition of large-scaled volumes of imaging data needed to produce the most successful computer algorithms. In response, the British Society of Thoracic Imaging research network in partnership with the National Health Service in the UK created a repository of chest radiographs, CT 
scans, and magnetic resonance imaging images. The goal is to join with other international entities to make data available to research and commercial groups to accelerate technological innovations designed to improve COVID-19 patient care. Despite the potential, there are several limitations to the widespread use of AI to combat COVID-19. Firstly, we found no evidence of improved outcomes over standard provider-based patient care. Second, use in resource-limited areas is challenging. Third, there are potential ethical concerns using AI to make patient care decisions [132, 133]. An early systematic review of COVID-19-related prediction models published in the British Medical Journal by Wynants et al. reported underwhelming results [134]. Upon review of 107 studies of 145 models (91 diagnostic, 50 prognostic), the authors did not recommend any prediction models for use in current practice. They offer a detailed analysis, but important limitations included a high risk of bias, non-representative selection of control patients, and vague reporting of study population or intended use of the models. Chen and See also performed a review published in the JMIR and found several similar limitations [135]. In July of 2020, Bakker et al. from the Netherlands published a systematic review of the health and economic impact of big data analytics for clinical decision making (not specific to COVID-19) in the JAMIA [136]. They reviewed 12,311 papers for eligibility and found only seven papers that reported cost-savings and improved outcomes from implemented "big data analytics" models. They discussed validation and deployment costs as an important barrier to implementing models into clinical practice and a lack of consistent definitions of big data. Bakker et al. concluded that the high expectations of big data and AI systems have not yet been realized. However, we remain optimistic for the potential of $\mathrm{AI}$ in diagnosis, public health, clinical decision-making, and therapeutics. Improved data sharing to expand and diversify data sources, increased focus on translational research in diagnostic and predictive analytics, and the expanding role of clinical informaticists due to the pandemic can increase the usability of such systems in the future.

\section{Data Sharing and Interoperability}

The prevalence of the EHR creates the potential for timely health information exchange (HIE) that can enhance both clinical care of shared patients and enable the aggregation of big data. For example, the effectiveness of the AI systems described in the previous section is reliant on the size and quality of the datasets upon which they are built. Plasek et al. in JAMIA offer a perspective on the importance of cross-border data sharing during the COVID-19 pandemic and the use of diverse and heterogenous datasets in order to overcome inherent limitations of localized data sources [137]. Their article highlights that critical public health decision making, clinical research, and pharmaceutical development all rely on global data, coordination, and solidarity. Zeng et al. provide a review of classification systems, taxonomies, ontologies, and subject headings used during the COVID-19 pandemic [138]. Proper use of these tools are essential to support HIE and information management. Unified classification systems such as the ICD are critical in providing a common language for reporting and monitoring disease-related outcomes. Done correctly, aggregating big data across populations, across geographic boundaries, and over time can enable a better understanding of the novel coronavirus, intelligent predictions, and effective tracking and surveillance of populations. For more than a decade, common data models (CDMs) have been developed in healthcare with the intent of standardizing and facilitating HIE. One example of a large, commonly used CDM is the Observational Medical Outcome Partnership (OMOP), a public-private partnership founded by the US Foundation for the National Institute and now maintained by the Observational Health Data Sciences and Informatics consortium $[139,140]$. OMOP enables large-scale comparative effectiveness and safety evaluation across millions of patients [141]. And in fact, several observational studies of patients with COVID-19 have been performed based on OMOP or similar CDMs [142-146]. However, despite substantial efforts to achieve cohesive HIE, highly effective data sharing remained elu- sive when the COVID-19 pandemic started. As a result, several new large data consortia initiatives emerged specifically in response to COVID-19 [147], including the National COVID-19 Cohort Collaborative (N3C, Haendel et al. [148]), the Consortium for Clinical Characterization of COVID-19 by EHR (4CE, Brat et al. [149]), and the Secure Collective Research (SCOR, Raisaro et al. [147]). None are universally adoptable though each offers potential for collaborative learning and eventual clinical translation. In addition, there are examples of smaller initiatives and tools developed to enhance interoperability of health systems. Dong et al. created a normalization system for mapping heterogenous locally developed COVID-19 testing nomenclature to the international standard Logical Observation Identifiers and Codes (LOINC) codes [150], termed COVID-19 TestNorm [151]. With an accuracy of $97.4 \%$, the authors report that widespread use of the tool can facilitate data aggregation and research. To aid local, national and international HIE across all stakeholders, the US CDC developed the CDC COVID-19 Information Management Repository [152]. Garcia et al. in JAMIA provided a thorough introduction to the in-depth repository [153]. The website supported by the CDC coalesces information from national organizations such as the Council of State and Territorial Epidemiologists and the Office of the National Coordinator for Health Information as well as international organizations such as the WHO, LOINC, ICD-10, and Systematized Nomenclature of Medicine (SNOMED). The repository categorizes COVID-19 data interoperability information into the following sections, among others: General resources: regulatory guidance documents, data sources, foundational semantic interoperability standards; Representing healthcare data for emergency medical services; Patient's clinical encounter: standard codes for diagnoses, clinical procedures, billing, and death certificates; COVID-19 public health reporting; Laboratory data exchange and laboratory surveillance: codes for COVID-19 specimen submission, test orders, test results; and geospatial data sets and reference sources.

However, the global infrastructure for HIE is still lacking and gaps in interoperability and data sharing have long been 
challenging. Cosgriff et al. emphatically state in The Lancet Digital Health, "never before has the failure to build robust data sharing systems for large-scale near real-time analysis in health care been more obvious" [154]. There is no single publicly available, multi-national registry containing patient-level COVID-19 data that can be vigorously studied and used to develop highly predictive models or answer potential clinical questions. Similarly, a recent study by Bruthans in IJMI found that the cross-border interoperability of electronic prescribing systems in the European union and USA is limited, directly affecting the efficiency of healthcare systems [155]. O'Reilly-Shah et al. in Anesthesia \& Analgesia comment on this shortcoming and describe several forces that undermine efforts to improve interoperability [156]. Counterincentives of EHR vendors, lack of standardized specifications, and privacy and ethical concerns are among many reasons for persistent information silos. Furthermore, data collection and processing must be done responsibly and with respect for privacy and confidentiality [157]. Ienca and Vayena in Nature Medicine articulate how the failure to do so can violate individual privacy rights, override informed consent, and fuel distrust of the geopolitical and health systems [157]. This is no easy task as well-intentioned regulations can sometimes result in more harm than good. Lenert and McSwain discuss how federal regulations in the USA created to protect patients' rights have limited the flow of information across systems, negatively affecting patient care during the pandemic [158]. Operationally, inadequate interoperability significantly impacted the ability of providers and hospitals to effectively report confirmed cases of COVID-19 to public health agencies. An analysis of national health security capacities published in The Lancet by Kandel et al. found that only $76 \%$ of countries globally had a robust detection capacity [159]. Holgrem et al. in JAMIA discussed barriers to effective and timely reporting during the COVID-19 pandemic [160]. They surveyed American Hospital Association data fielded from chief executive officers of US hospitals to identify barriers to electronic submission of health information to public health agencies, including public health agencies' inability to electronically receive data (reported by $41.2 \%$ of hospitals), interface-related costs $(31.9 \%)$, difficulty extracting EHR data $(14.7 \%)$, varied vocabulary standards $(14.2 \%)$, inability of hospitals to send data electronically (8.3\%), and not knowing where to send data (3.3\%). The finding that $40 \%$ of public health departments required manual reporting highlights that investment in technology and interoperability infrastructure is necessary for highly functioning CIS. Stenner et al. contributed a viewpoint in ACI making a distinct yet important point that more information is not always beneficial [161]. They describe how overloading the information receiver with unfiltered electronic health information can present more data than can be reasonably processed. The result may be a paradoxical decrease in efficiency and the ability to find clinically relevant data points. Foraker et al. discuss the well-known challenges to effective data-sharing [162]. The authors offer a framework to address inadequate infrastructure, duplication of data requests, and insufficient coordination by responsible entities: (1) Identify and fill technology gaps relevant to data sharing efforts in order to reduce the time to implementation; (2) Engage in the collaborative design of data-sharing requirements and transmission mechanisms to reduce redundancies and establish economies of scale; (3) Facilitate cross-domain discussion involving legal and research compliance to identify pathways for data-sharing efforts to be appropriately and effectively managed from a regulatory perspective; and (4) Establish or participate in multi-institutional convening or coordinating activities. Sim et al. in Science called for the US National Institutes of Health (NIH) to take the lead on achieving "findable, accessible, interoperable, and reusable" scientific data sharing [163]. As the largest global funder of biomedical research, the authors argue for NIH policy to include mandatory data sharing beginning with any registered clinical trial. Given the variability in international healthcare delivery systems and governance of health IT infrastructure, development and implementation costs, language barriers, and other misaligned incentives, it stands to reason that truly effective HIE will be a challenge for the next decade and beyond. Though one single international system seems unlikely, the trend in collaborative efforts focused on standardized data collection and nomenclature remain encouraging.

\section{Epidemiologic Reporting}

A core foundational tenet in the effective management of any disease is the ability to have timely and accurate data around underlying structural factors, processes and outcomes. Similarly, management of a pandemic requires large-scale data across both time and space. Many academic organizations, private institutions, and governmental agencies have developed geographical information systems (GISs) -- largely in the form of online dashboards -- to enable real-time epidemic monitoring and subsequent clinical responses for COVID-19. The first and most notable global COVID-19 dashboard came from Lauren Gardner and her team at the Center for Systems Science and Engineering at John's Hopkins University (USA) [164]. They developed an interactive web-based dashboard to visually display and track confirmed cases, deaths, and recoveries from COVID-19 at the national, provincial, or city level depending on the country of interest [164]. They adopted a semi-automated living data stream strategy aggregating data from multiple authoritative sources including the WHO, regional public health departments and respective CDCs, as well as social media, news feeds, and direct communications. First publicly shared on January $22^{\text {nd }}, 2020$, the real-time COVID-19 dashboard was able to report newly infected cases ahead of the WHO. It quickly became a widely cited, highly reliable source of data for academics, public health authorities, and the general public at large. Several other well-known COVID-19 trackers have been developed and made available including the WHO Coronavirus (COVID-19) Dashboard, the CDC COVID-19 Data Tracker, and HealthMap [165-167]. Kamel Boulos and 
Geraghty in the International Journal of Health Geographics wrote an early editorial outlining how these and other GISs can support the fight against infectious disease outbreaks through data transparency, communication, and by creating a shared understanding of the pandemic [168]. There are limits to the level of granularity of data able to be displayed within global GISs, as well as limits around regional and other needed micro-analyses. As such, there was a need for additional sources of information to assist local municipalities. In one example from the USA, Wissel et al. created an open access interactive dashboard that aggregated data on a county-level to display temporal changes in testing capacity, cases, and deaths [169]. Similarly, CovidCounties, a COVID-19 County Tracker from the Butte Lab and OptumLabs (USA), was developed and published online [170]. In many instances, local public health departments and health systems created their own dashboards based on automatic, semi-automatic, or manually reported case data $[15,19,171]$. This locally derived data was frequently integrated with local EHR instances to give administrators and front-line providers real-time information to guide clinical decisions. In another intriguing application of visual reporting, Thorlund et al. developed a COVID-19 clinical trials registry utilizing AI to supplement identification of qualified trials [172]. They then mapped trials based on location, patient, and intervention characteristics to create a visual dashboard of global COVID-19 research efforts. Dixit et $a l$. in JAMIA published the development of eVisit Operations Dashboards for executives, telehealth operational leaders, and management teams at a large health system in the USA [173]. They share several lessons regarding rapid implementation of visual reporting displays: use a lightweight and iterative user-centered approach; basic (accurate) information is better than no visualization; clearly indicate development status and timeliness; develop prioritization criteria for visualization and feature development; stabilize the data environment as much as possible; and metric design, user literacy, and user control should be based on audience.

\section{Digital Contact Tracing and Exposure Notification Systems}

Contact tracing, a key component of minimizing disease transmission and essential tactic of pandemic management consists of identification, notification, and subsequent surveillance of individuals who have been exposed [174, 175]. Conventional measures of contact tracing, largely based around manual interviews, are laborious and time-consuming and have been challenging to do effectively during the COVID-19 pandemic [176]. Furthermore, manual tracing is prone to recall error from interviewed patients. Overwhelmed with new cases, many organizations explored technology-based tools to create automatic or partially automatic digital contact tracing [177]. As described above, Taiwan was one country with a robust mechanism of digital contact tracing that putatively helped to reduce transmission [14, 98]. In an early publication in Science, Ferretti et al. utilized mathematical modelling to demonstrate that algorithmic, immediate notifications to exposed individuals with a contact-tracing mobile phone application could contain the spread of COVID-19, especially if used in concert with physical distancing [177]. The beneficence of electronic contact allows for quick identification of close contacts of positive cases followed by rapid testing and quarantine of suspect or positive cases to contain the spread of the virus [178]. Several countries, including Singapore, South Korea, and Australia quickly developed electronic contact tracing applications and processes [179, 180]. Using Bluetooth technology or Global Positioning Systems, mobile phones can securely track an individual's proximity to other mobile devices that are using the same technology. Should one of those individuals become infected with COVID-19, it would be recorded in either a centralized or decentralized database and an automatic notification would be sent to the exposed individual with applicable instructions to self-quarantine. Online reports indicate some success of these applications. For example, Singapore reported that as of November 3, 2020, their contact tracing application had helped identi- fy 25,000 close contacts of COVID-19 cases, of which 160 tested positive [181]. Dozens of nations have now published these smart phone-based digital contact tracing applications [182]. However, we found no reports of successful widespread implementation of such a strategy. Huang et al. in JMIR Mhealth Uhealth compared the effectiveness of the Bluetooth-based TraceTogether contact tracing app with a wearable tag real-time locating system (RTLS) in the National Centre for Infectious Diseases, the national referral center for COVID-19 screening in Singapore [183]. They found that RTLS had a higher sensitivity of $95.3 \%$ vs $6.5 \%$ for detecting patient contacts. The authors discussed that while wearable RTLS is more effective in a healthcare setting, deployment and implementation would be substantially more challenging in a community setting. With increased penetration of the app and token in the country, officials hope to see updated figures in the near future. Two systematic reviews were conducted assessing the effectiveness of digital contact tracing. Braithewaite et al. identified 4,036 studies and included 15 studies in their final analysis. They found no epidemiological studies that offered empirical evidence comparing automated with manual contact-tracing systems [184]. Anglemeyer et al. performed a Cochrane review and concluded that the effectiveness of digital solution in contact tracing is largely unproven [185]. There are several important reasons why this strategy has not widely succeeded in practice. First, effective transmission prevention during a pandemic would require that most of the population has access to smart phones. At the beginning of 2020, $46.4 \%$ of the world population had no access to the internet [186]. This perhaps surprising figure highlights that digital contact tracing and many of the technologies described in this survey serve mainly to benefit a relatively privileged segment of society. Furthermore, to effectively impact COVID-19 transmission, various models suggest end-user adoption thresholds between $56 \%$ to $95 \%$, accurate self-reporting, and adherence to home isolation notifications of 90 to $100 \%$ are necessary [184]. End-user compliance however, is largely voluntary and thus presents major barriers to effectiveness. Finally, digital contact tracing 
poses privacy concerns related to accessing personal information [101]. Most app developers have taken considerable measures to encrypt data, delete after its useful time, or otherwise protect confidentiality [180]. However, even perceived potential to expose personal information substantially limits adoption, highlighting that trust between the government and the population needs to be present for electronic contact tracing to be successful. However, both Braithewate et $a l$. and Anglemeyer et al. reported the potential for partially or fully automated digital contact tracing to provide more complete contact identification and reduce the amount of time required to complete contact tracing $[184,185]$. Digital contact tracing has also been applied within hospitals and among healthcare workers [187]. By integrating data from five different hospital-based information systems, Venkataraman et al. rapidly and comprehensively tracked COVID-19 patients' activity within their system to identify both healthcare workers and patients at risk [188]. Staff were then able to focus time on interviews and risk assessment rather than contact identification. Following implementation of the algorithm, the authors observed a greater than $60 \%$ reduction in time needed to complete contact tracing. For most countries, reliance on digital contact tracing is perhaps unrealistic. However, incentive-based digital strategies can serve to augment rigorous manual contact tracing and increased access to digital technology among general populations could offer a more reliable strategy for outbreak management in the future.

\section{Outcomes from Clinical Information Systems on Non-COVID-19 Patient Care}

Providers and the healthcare delivery system in which they practice must ultimately strive and be held accountable for continually optimizing patient outcomes. This is reflected by improving disease specific outcomes, process outcomes, and minimizing medical errors and adverse events. It is too early to fully understand the role that CIS played in the morbidity and mortality of COVID-19 patients or the transmission of COVID-19 across populations. However, there is mounting evidence from the past decade examining how CIS are frequently used to improve patient outcomes in other areas of medicine. The opioid crisis is a recent example where frontline care providers and public health policy aligned with CIS to make meaningful improvements. Before COVID-19 emerged, the opioid crisis was the most prominent global epidemic with over 58 million reported users and over 350,000 deaths related to opioids worldwide $[189,190]$. CIS have been leveraged to support decision making around analgesia with opioid and non-opioid prescribing recommendations as well as to facilitate adherence to state-level requirements for prescription drug monitoring in all settings [191]. Weiner et al. in the Joint Commission Journal on Quality and Patient Safety report how a collaborative approach utilizing health IT reduced the flow of opioids into communities while increasing prescriptions for medication assisted treatment, key process measures [191]. Chronic opioid addiction is complex and observed reductions in opioid overdoses have been less consistent highlighting the need for continued advancement to translate process improvements to measures like mortality. In the inpatient setting, CIS have been leveraged to successfully influence provider behavior with the aim of improving clinical outcomes [192]. Emergency medicine and critical care medicine are two areas that have embraced the use of data and CIS to support management of persistently challenging diseases. However, the literature shows mixed results, a perfect example being sepsis. EHR-based tools have been credited with improvement in the prediction of sepsis and the appropriate and timely delivery of proven interventions such as intravenous fluids and antibiotics, yet other studies have shown limited clinical impact on sepsis treatment performance measures and overall mortality [193-195]. Similarly, implementation of non-EHR based care bundles to manage sepsis have shown no impact on mortality, highlighting that adherence to protocolized care strategies still has limitations among difficult-to-treat diseases [196]. There is ample evidence that clinical pathways and clinical guidelines are more likely to result in practice changes - usually determined by process measure compliance - if accompanied by effective integration into an EHR. For example, in the surgical areas, most hospital-based implementations of evidence-based clinical pathways have leveraged order sets embedded in the EHR to incorporate processes related to enhanced recovery and CDS alerts related to preventable harms [197]. Importantly, EHR modification alone may be effective at improving process measure compliance. However, this may not be sufficient for moving the needle on clinical outcomes like surgical site infection, venous thromboembolism and length of hospital stay [198]. Rather, EHR-based tools must be used in combination with thoughtful programmatic implementation and operational change management to meaningfully improve patient outcomes as evidenced by experience in colorectal, gynecologic and urologic surgery [199]. When considering vexing clinical problems, it is important to consider the role CIS can play in optimizing disease management, but this must be done in a deliberate manner that weighs both the technical and adaptive components of clinical care and provider behavior [200]. Convincingly, clinical informaticists, together with engaged leadership and empowered frontline providers, have helped to advance quality and patient safety along the continuum of healthcare before and during the COVID-19 pandemic.

\section{Discussion}

To effectively control the COVID-19 pandemic, the following global strategic objectives are advised by the WHO [201]:

- Case prevention across all sectors of government and society through hand hygiene, respiratory etiquette, and individual level physical distancing;

- Rapid and accurate case identification and isolation;

- Effective tracing, quarantining, and support of exposed individuals;

- Context appropriate infection prevention and control through population level physical distancing measures, and appropriate and proportionate travel restrictions; 
- Reduce mortality by providing appropriate clinical care, protecting frontline workers, and ensuring continuity of essential health services;

- Development and distribution of safe and effective vaccines and therapeutics.

In the current survey, we present examples from the literature demonstrating how CIS have supported these management strategies during the COVID-19 pandemic as the world eagerly awaits effective vaccines and therapeutics. CIS enabled or enhanced the implementation of health safety protocols; the ability to screen, test, and diagnose at scale; data collection, analysis and reporting; population cohorting and contact tracing; the rapid advancement of research; and the provision of safe and effective essential healthcare through telehealth services. Informatics has also been used in the search for effective therapeutics and vaccinations through predictive frameworks relying on immuno-informatics computational modeling [202-204]. In addition, informatics infrastructures such as immunization registries will be essential to the timely and equitable distribution of an effective vaccine [205, 206]. Thus, CIS and health informatics have been successfully utilized and will continue to be deployed across the spectrum to facilitate pandemic management [207].

Despite our optimism in the current capabilities of CIS to support the management of COVID-19, everyday healthcare, and yet unknown public health crises, there remain significant gaps in the field. Challenges persist in translating promising research into clinical practice in areas such as AI and predictive analytics [208, 209], achieving end-user adoption of tools such as digital contact tracing applications [185], addressing ethical and privacy concerns, and realizing impacts on clinical outcomes such as improved mortality following successes in process measures. Perhaps the most significant challenges to address are a lack of generalizability, interoperability and data sharing, and coordination among healthcare organizations, public health agencies, and other healthcare entities.

Unlike a novel disease-altering medication or a superior anti-hypertensive that can be dispensed to pharmacies and patients around the world, proven EHR-based interventions are rarely shared across health systems or internationally [210]. Herein, we discuss several use cases of CIS-based tools that can substantially improve processes and patient care when facing a pandemic. However, the generalizability of most is quite limited as end-user adoption requires system-wide CIS development, accommodation for local workflows, and programmatic implementation. Informaticists from different healthcare organizations are persistently re-inventing the wheel, developing systems and configuring EHRs repeatedly to address what are most often shared challenges. Similarly, effective HIE and big data sharing are challenging due to varied data definitions and collection, lack of interoperability between EHRs, and uncoordinated efforts among stakeholders [11]. Even large health systems cannot fully achieve the potential of CIS alone. Madhavan et al. in JAMIA offer a thorough perspective on the issue of uncoordinated responses outlining the need to engage stakeholders from the informatics, public health, epidemiologic, and administrative communities in public, private, and non-profit sectors to strive for standardization and collaboration [211]. Among many others, these authors submit a call to action to unify, simplify, standardize, and share.

Since the onset of the pandemic, research articles have been published via open access, GIS dashboards have been shared publicly, algorithms and codes have been posted online for others to use, and multiple large data consortiums have re-emerged in an effort to collaborate globally in the fight against COVID-19 [124, 131, 147, 177]. It remains to be seen whether the widely prevalent intentions to equitably share and standardize information technology will prevail. Time will tell if lessons learned from COVID-19 allow a more coordinated and potentially more effective informatics response to the next pandemic. Certainly, substantially more informatics infrastructure has been developed in the past several months when compared to the decades following previous infectious disease outbreaks. However, we suspect coordination and generalizability will be significant challenges for the informatics community for the foreseeable future.
In the face of these challenges, CIS and clinical informaticists made prominent and influential contributions in the global response to the COVID-19. Through the timely and effective distribution of reliable information, CIS can enhance patient care during a public health crisis.

\section{References}

1. Zhu N, Zhang D, Wang W, Li X, Yang B, Song J, et al. A Novel Coronavirus from Patients with Pneumonia in China, 2019. N Engl J Med 2020;382:727-33.

2. Organization WH. Coronavirus Disease 2019 (COVID-19) Situation Report - 52 . 2020 [cited 2020 Sep 27][https://apps.who.int/iris/ bitstream/handle/10665/331685/nCoVsitrep01Apr2020-eng.pdf]

3. COVID-19 Dashboard by the Center for Systems Science and Engineering (CSSE) at Johns Hopkins University (JHU) [cited 2020 Nov 30] [https://coronavirus.jhu.edu/map.html]

4. Bedford J, Enria D, Giesecke J, Heymann DL, Ihekweazu C, Kobinger G, et al. COVID-19: towards controlling of a pandemic. Lancet 2020;395(10229):1015-8.

5. Wu Z, McGoogan JM. Characteristics of and Important Lessons From the Coronavirus Disease 2019 (COVID-19) Outbreak in China: Summary of a Report of 72314 Cases From the Chinese Center for Disease Control and Prevention. JAMA 2020;323(13):1239-42.

6. Gates B. Responding to Covid-19 - A oncein-a-century pandemic? N Engl J Med. 2020;382(18):1677-9.

7. COVID-19 Open Research Dataset [Internet]. The Semantic Scholar team at the Allen Intitute for AI. [cited 2020 Nov 29] [https://www.semanticscholar.org/cord19]

8. Bakken S. Informatics is a critical strategy in combating the COVID-19 pandemic. J Am Med Inform Assoc 2020;27(6):843-4.

9. Legido-Quigley H, Mateos-García JT, Campos VR, Gea-Sánchez M, Muntaner C, McKee M. The resilience of the Spanish health system against the COVID-19 pandemic. Lancet Public Health 2020;5(1):e251-2.

10. Gostin LO, Hodge JG. US Emergency Legal Responses to Novel Coronavirus: Balancing Public Health and Civil Liberties. JAMA 2020;323(12):1131-2.

11. Jones BA, Sergejeff K, Sherriff A, Teevan C, Veron $\mathrm{P}$. The challenge of scaling up the European Union's global response to COVID-19. ECDPM Making Policies Work; 2020.

12. Omer SB, Malani P, Del Rio C. The COVID-19 Pandemic in the US: A Clinical Update. JAMA 2020;323(18):1767-8.

13. Sittig DF, Singh H. COVID-19 and the Need for a National Health Information Technology Infrastructure. JAMA 2020;323(23):2373-2374.

14. Wang $\mathrm{CJ}, \mathrm{Ng} \mathrm{CY}$, Brook RH. Response to COVID-19 in Taiwan. JAMA 2020;323(14):1341-2. 
Reeves et al.

Table 1 Selected articles for detailed review.

\begin{tabular}{|c|c|c|c|c|c|}
\hline $\begin{array}{l}\text { Survey } \\
\text { Section }\end{array}$ & Reference & Author(s) & Country & Journal & Key Contribution(s) \\
\hline \multirow{8}{*}{ 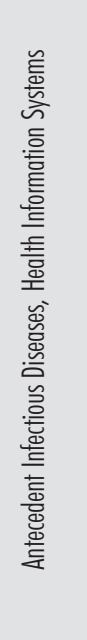 } & 28 & Zhao et al. & China & Telemed e-Health & $\begin{array}{l}\text { Description of Chinese construct of regional and national informatics } \\
\text { systems in response to SARS }\end{array}$ \\
\hline & 29 & Chen et al. & Taiwan & Information Systems Research & $\begin{array}{l}\text { Identifies framework for detecting emerging infectious disease with } \\
\text { central and local coupling and decoupling circles }\end{array}$ \\
\hline & 30 & Mandl et al. & USA & JAMIA & $\begin{array}{l}\text { Syndromic surveillance systems can be used for detection of infectious } \\
\text { diseases }\end{array}$ \\
\hline & 33 & Advani et al. & USA & $\begin{array}{l}\text { Studies in health technology } \\
\text { and informatics }\end{array}$ & Created interactive dashboard for local municipalities across the US. \\
\hline & 34 & Keck et al. & USA & JAMIA & $\begin{array}{l}\text { Developed EHR-based influenza surveillance system for American } \\
\text { Indian and Alaska Native populations }\end{array}$ \\
\hline & 37 & Landman et al. & USA & $\begin{array}{l}\text { Disaster Medicine and Public } \\
\text { Health Preparedness }\end{array}$ & Used CDS to ensure screening for Ebola \\
\hline & 43 & Borycki et al. & $\begin{array}{l}\text { Canada, Australia, } \\
\text { USA, Finland, Japan }\end{array}$ & $\begin{array}{l}\text { Yearbook of Medical } \\
\text { Informatics }\end{array}$ & $\begin{array}{l}\text { Review of literature regarding health information technology used for } \\
\text { patient-centered care during Ebola outbreak. }\end{array}$ \\
\hline & 44 & Mandl & USA & JAMA & Viewpoint on EHR as public health tool for outbreaks of infectious disease \\
\hline \multirow{8}{*}{ 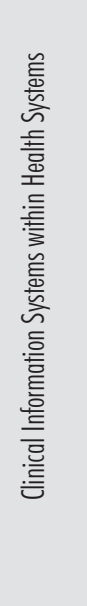 } & 19 & Reeves et al. & USA & JAMIA & $\begin{array}{l}\text { Rapid implementation of EHR-based tools used to support clinical } \\
\text { patient care during COVID-19 pandemic }\end{array}$ \\
\hline & 48 & Grange et al. & USA & $\mathrm{ACl}$ & $\begin{array}{l}\text { Rapid rollout of Information Technology Services to support clinical } \\
\text { response to COVID-19. }\end{array}$ \\
\hline & 49 & Lin et al. & USA & JAMIA & $\begin{array}{l}\text { Informatics tools used for rapid onboarding of physician/staff and } \\
\text { enhanced communication with families }\end{array}$ \\
\hline & 50 & Milenkovic et al. & Serbia & IJMI & Created Al-driven patient triage and scheduling modules \\
\hline & 51 & Yan et al. & China & JAMIA & $\begin{array}{l}\text { Analyzed hospital webpages in China to determine themes of Health IT } \\
\text { use in mainland China }\end{array}$ \\
\hline & 52 & Ye et al. & China & JMIR & $\begin{array}{l}\text { Provide broad framework of how information technology is used in } \\
\text { mainland China }\end{array}$ \\
\hline & 53 & Sylvestre et al. & France & JAMIA & Responding to pandemic without an EHR \\
\hline & 54 & Kannampallil et al. & USA & JAMIA & $\begin{array}{l}\text { Describe transition of informaticist from academic to operational leader } \\
\text { and share lessons learned }\end{array}$ \\
\hline \multirow{6}{*}{ 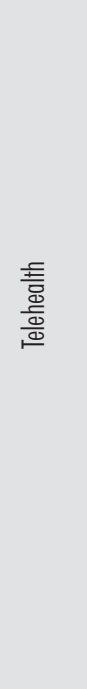 } & 55 & Hong et al. & USA & $\begin{array}{l}\text { JMIR Public Health and } \\
\text { Surveillance }\end{array}$ & $\begin{array}{l}\text { Correlation between COVID-19 cases and telehealth internet search } \\
\text { volume; Survey of telehealth adoption and tele-ICU capabilities in US } \\
\text { hospitals prior to COVID-19 }\end{array}$ \\
\hline & 57 & Bhaskar et al. & $\begin{array}{l}\text { Australia, Canada, Ka- } \\
\text { zakhstan, Trinidad and } \\
\text { Tobago, USA, United } \\
\text { Kingdom, Ireland, } \\
\text { Israel, Philippines, } \\
\text { India, and Poland }\end{array}$ & Frontiers in Public Health & $\begin{array}{l}\text { Telemedicine status pre and post COVID-19 outbreak in countries } \\
\text { around the world with recommendations for further development }\end{array}$ \\
\hline & 63 & Mehrotra et al. & USA & NEJM Catalyst & $\begin{array}{l}\text { Early account of rapid telehealth roll-out in US with heavy reliance on } \\
\text { telephone encounters }\end{array}$ \\
\hline & 65 & Mann et al. & USA & JAMIA & $\begin{array}{l}\text { Description of rapid rollout of video visits in urgent care and ambulato- } \\
\text { ry clinic settings at the onset of the pandemic in New York }\end{array}$ \\
\hline & 70 & $\begin{array}{l}\text { Monaghesh \& } \\
\text { Haijzadeh }\end{array}$ & Iran & BMC Public Health & $\begin{array}{l}\text { Systematic review of telehealth utilization during COVID-19 outbreak } \\
\text { from Dec } 2019 \text { to April } 2020\end{array}$ \\
\hline & 73 & Wosik et al. & USA & JAMIA & $\begin{array}{l}\text { Description of phases of telehealth transformation during COVID-19 in } \\
\text { ambulatory and inpatient settings }\end{array}$ \\
\hline
\end{tabular}


Table 1 continued Selected articles for detailed review.

\begin{tabular}{|c|c|c|c|c|c|}
\hline $\begin{array}{l}\text { Survey } \\
\text { Section }\end{array}$ & Reference & Author(s) & Country & Journal & Key Contribution(s) \\
\hline \multirow{6}{*}{ 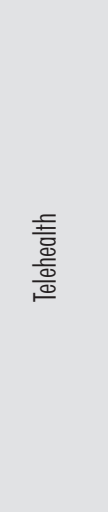 } & 75 & Vilendrer et al. & USA & JAMIA & $\begin{array}{l}\text { Inpatient utilization of telemedicine across three different health systems } \\
\text { in California in response to the pandemic }\end{array}$ \\
\hline & 74 & Hron et al. & USA & $\mathrm{ACl}$ & $\begin{array}{l}\text { Rapid implementation of an inpatient telehealth program in an academic } \\
\text { children's hospital with significant utilization volumes }\end{array}$ \\
\hline & 76 & Ong et al. & USA & $\mathrm{ACl}$ & $\begin{array}{l}\text { Description of implementation of inpatient telehealth program in an } \\
\text { academic medical center in response to COVID-19 with delineation of } 13 \\
\text { use cases and } 8 \text { device options }\end{array}$ \\
\hline & 77 & Jones et al. & USA & $\begin{array}{l}\text { Diabetes Technology \& } \\
\text { Therapeutics }\end{array}$ & $\begin{array}{l}\text { Demonstration of unchanged glycemic measures as outcome metric in pre-- } \\
\text { post- intervention comparison of virtual diabetes program during COVID-19 }\end{array}$ \\
\hline & 78 & Wijesooriya et al. & Netherlands, USA & Ped Resp Reviews & Applications of telehealth in medical education and clinical research \\
\hline & 91 & Nouri et al. & USA & NEJM Catalyst & $\begin{array}{l}\text { Demonstration of statistically significant exacerbations of healthcare } \\
\text { access inequity during COVID-19 pandemic }\end{array}$ \\
\hline \multirow{8}{*}{ 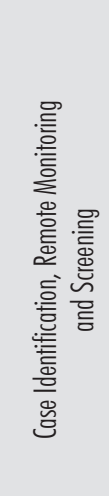 } & 14 & Wang et al. & Taiwan & JAMA & $\begin{array}{l}\text { Description of Taiwanese national response to pandemic utilizing technol- } \\
\text { ogy for rapid case identification, contact tracing, and surveillance }\end{array}$ \\
\hline & 104 & Judson et al. & USA & JAMIA & Created a self-triage and scheduling tool \\
\hline & 105 & Judson et al. & USA & JAMIA & Digital chatbot used for daily screen of healthcare employees \\
\hline & 106 & Annis et al. & USA & JAMIA & $\begin{array}{l}\text { Remote patient monitoring system used to manage COVID-19 symptoms } \\
\text { at home }\end{array}$ \\
\hline & 103 & Ford et al. & USA & JAMIA & $\begin{array}{l}\text { Used biomedical informatics tools for remote monitoring, biosensors, and } \\
\text { dashboard }\end{array}$ \\
\hline & 107 & Perlman et al. & USA, Israel, Great Britain & JMIR & Al drive self-assessment, symptom checker \\
\hline & 108 & Perez-Alba et al. & Mexico & JAMIA & Onsite electronic self-administered triage tool \\
\hline & 109 & Turer et al. & USA & JAMIA & Electronic personal protective equipment through use of telehealth \\
\hline 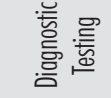 & 115 & Weemaes et al. & Belgium & JAMIA & $\begin{array}{l}\text { Laboratory information systems can help alleviiate bottlenecks in } \\
\text { COVID-19 diagnostic testing }\end{array}$ \\
\hline \multirow{15}{*}{ 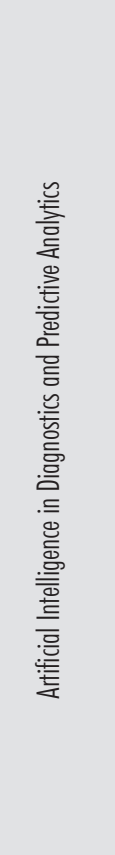 } & 123 & Debnath et al. & USA & Bioelectronic Medicine & $\begin{array}{l}\text { Al systems can augment clinical decisions in diagnosis and screening, rish } \\
\text { stratification, prognosis, and allocation of resources }\end{array}$ \\
\hline & 111 & Obeid et al. & USA & JAMIA & Natural language processing in a virtual care screening tool \\
\hline & 124 & Zhang et al. & China & Cell & Al utilizing CT images can aid in diagnosis/prognosis \\
\hline & 125 & Li et al. & China & Radiology & Al utilizing (Ti images can aid in diagnosis \\
\hline & 126 & Murphy et al. & Netherlands & Radiology & Al for diagnostics in chest XR images \\
\hline & 127 & Hurt et al. & USA & Journal of Thoracic Imaging & Al for diagnostic imaging implemented as cloud-based tool \\
\hline & 128 & Carlile et al. & USA & JACEP Open & $\begin{array}{l}86 \% \text { of ED physicians found an Al tool was easy to use in workllow and } \\
20 \% \text { reported algorithm impacted clinical decision making }\end{array}$ \\
\hline & 129 & $\begin{array}{l}\text { Goodman-Meza } \\
\text { et al. }\end{array}$ & USA & Plos One & Al utilizing ancillary lab values can aid in diagnosis/screening \\
\hline & 116 & Mei et al. & USA and China & Nature Medicine & Al as useful triage tool while definitive PCR tests result \\
\hline & 112 & Liv et al. & China & JMIR & Al tool for CDS among general practitioners \\
\hline & 130 & Liang et al. & China & Nature Com & Deep-learning neural network to predict critical illness of COVID-19 patients \\
\hline & 131 & Jacob et al. & United Kingdom & European Respiratory Journal & National COVID-19 Chest imaging Database in the United Kingdom \\
\hline & 134 & Wynants et al. & Europe & British Medical Journal & Systematic review found no Al models recommended for use in clinical practice \\
\hline & 135 & Chen and See & Singapore & JMIR & Systematic review highlighting shortcomings without definitive condusion \\
\hline & 136 & Bakker et al. & Netherlands & JAMIA & $\begin{array}{l}\text { Systematic review of health and economic impact of big data analytics } \\
\text { found benefit no definitive benefit in current literature }\end{array}$ \\
\hline
\end{tabular}


Reeves et al.

Table 1 continued Selected articles for detailed review.

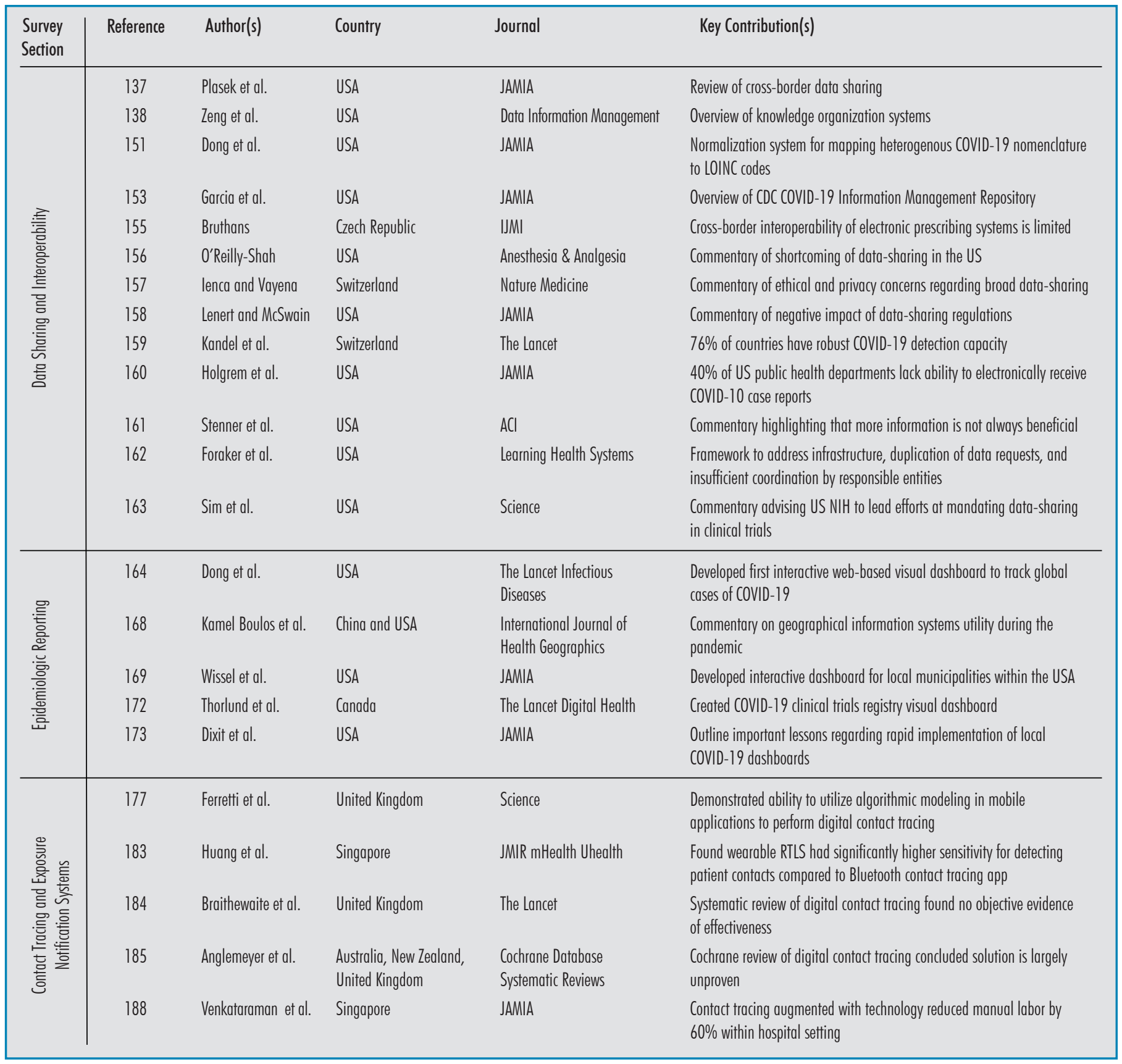

Abbreviations: SARS = Severe Acute Respiratory Distress Syndrome; USA = United States of America; US = United States; JAMIA = Journal of the American Medical Informatics Association; EHR = electronic health record; CDS = clinical decision support; JAMA = Journal of the American Medical Association; COVID-19 = Coronavirus Disease 2019; Al = Arrificial Intelligence; JMIR = Journal of Medical Internet Research; IT = Information Technology; ICU = intensive care unit; NEJM = New England Journal of Medicine; $C T=$ computed tomography; XR = X-ray; JACEP = Journal of the American College of Emergency Physicians; ED = Emergency Department; PCR = polymerase chain reaction; LOINC = Logical Observation Identifiers Names and Codes; $C D C=$ Centers for Disease Control and Prevention; $\mathrm{NIH}=$ National Institutes of Health; RTLS $=$ real-time locating system 
15. Ronquillo JG, Lester WT, Zuckerman DM. Using informatics to guide public health policy during the COVID-19 pandemic in the USA. J Public Health (Oxf.) 2020;42(4):660-4.

16. Budd J, Miller BS, Manning EM, Lampos V, Zhuang M, Edelstein M, et al. Digital technologies in the public-health response to COVID-19. Nat Med 2020;26(8):1183-92.

17. Combi C, Pozzi G. Clinical Information Systems and Artificial Intelligence: Recent Research Trends. Yearb Med Inform 2019;28(1):83.

18. Islam MM, Poly TN, Li YCJ. Recent Advancement of Clinical Information Systems: Opportunities and Challenges. Yearb Med Inform 2018;27(1):83.

19. Reeves JJ, Hollandsworth HM, Torriani FJ, Taplitz R, Abeles S, Tai-Seale M, et al. Rapid Response to COVID-19: Health Informatics Support for Outbreak Management in an Academic Health System. J Am Med Inform Assoc 2020;27(6):853-9.

20. Hollander JE, Carr BG. Virtually Perfect? Telemedicine for Covid-19. N Engl J Med 2020;382(18):1679-81.

21. Keesara S, Jonas A, Schulman K. Covid-19 and Health Care's Digital Revolution. N Engl J Med 2020;382(23):e82.

22. The Office of the National Coordinator for Health Information Technology. Annual Update to the Adoption of a Nationwide System for the Electronic Use and Exchange of Health Information 2018 Report Congress. [https://www.healthit. gov/sites/default/files/page/2018-12/2018-HITECH-report-to-congress.pdf]

23. Development $\mathrm{O}$ for $\mathrm{EC}$ and. Health at a Glance: Europe 2020 state of health in the EU cycle. OECD Publishing; 2020. [https://www.oecd-ilibrary.org/social-issues-migration-health/healthat-a-glance-europe-2020_82129230-en]

24. World Health Organization. Global diffusion of eHealth: making universal health coverage achievable. Report of the third global survey on eHealth. Global Observatory for eHealth; 2016. [https://www.who.int/goe/publications/ global_diffusion/en/]

25. Leung GM, Medley AJ, Ho LM, Chau P, Wong IOL, Thach TQ, et al. The epidemiology of severe acute respiratory syndrome in the 2003 Hong Kong epidemic: An analysis of all 1755 patients. Ann Intern Med 2004;141(9):662-73.

26. Fidler DP. Emerging trends in international law concerning global infectious disease control. Emerg Infect Dis 2003;9(3):285.

27. Hughes JM. The SARS Response - Building and Assessing an Evidence-Based Approach to Future Global Microbial Threats. JAMA 2003;290(24):3251-3.

28. Zhao J, Zhang Z, Guo H, Li Y, Xue W, Ren $\mathrm{L}$, et al. E-health in China: Challenges, initial directions, and experience. Telemed J E Health 2010;16(3):344-9.

29. Chen Y Da, Brown SA, Hu PJH, King CC, Chen $\mathrm{H}$. Managing emerging infectious diseases with information systems: Reconceptualizing outbreak management through the lens of loose coupling. Inf Syst Res 2011;22(3):447-68.

30. Mandl KD, Overhage JM, Wagner MM, Lober WB, Sebastiani P, Mostashari F, et al. Implement- ing Syndromic Surveillance: A Practical Guide Informed by the Early Experience. J Am Med Inform Assoc 2004;11(2):141-50.

31. Centers for Disease Control and Prevention. The 2009 H1N1 Pandemic: Summary Highlights, April 2009-April 2010 [cited 2020 Jan 10][https://www.cdc.gov/flu/pandemic-resources/2009-h1n1-pandemic.html]

32. Advani A, Turuvekere AM, Liu C, Rubin K, Lamer C, Cullen T. Design and assessment of a common, multi-national public health informatics infrastructure to enable H1N1 influenza surveillance. Stud Health Technol Inform 2010;160(Pt 1):452-6.

33. Senathirajah, Yalini; Kaufman, David; Bakker S. When speed is essential: Rapid configuration of a user-configurable 'web 2.0' based EHR for H1N1 decision support. International symposium on human factors engineering in health informatics, Medical Informatics Europe, Trondheim, Norway; 2011

34. Keck JW, Redd JT, Cheek JE, Layne LJ, Groom A V., Kitka S, et al. Influenza surveillance using electronic health records in the American Indian and Alaska Native population. J Am Med Inform Assoc 2014;21(1):132-8.

35. Indian Health Service. FACT SHEET: IHS Profile [Internet]. The Federal Health Program for American Indians and Alaska Natives; 2020 [cited 2020 Oct 1][https://www.ihs.gov/newsroom/factsheets/ihsprofile/]

36. Centers for Disease Control and Prevention 2014-2016 Ebola Outbreak in West Africa [Internet]. 2014 [cited 2020 Oct 10][https://www. cdc.gov/vhf/ebola/history/2014-2016-outbreak/ index.html]

37. Landman AB, Goralnick E, Teich JM. Clinical Decision Support in the Management of Patients with Suspected Ebola Infection. Disaster Med Public Health Prep 2015;5:591-4.

38. Oza S, Jazayeri D, Teich JM, Ball E, Nankubuge PA, Rwebembera J, et al. Development and deployment of the OpenMRS-Ebola electronic health record system for an Ebola treatment center in Sierra Leone. J Med Internet Res 2017;19(8):e294

39. Wesolowski A, Buckee CO, Bengtsson L, Wetter E, Lu X, Tatem AJ. Commentary: Containing the Ebola Outbreak - the Potential and Challenge of Mobile Network Data. PLoS Curr 2014;6:ecurrents.outbreaks.0177e7fcf52217b8b634376e$2 \mathrm{f3efc5e}$.

40. Danquah LO, Hasham N, MacFarlane M, Conteh FE, Momoh F, Tedesco AA, et al. Use of a mobile application for Ebola contact tracing and monitoring in northern Sierra Leone: A proof-ofconcept study. BMC Infect Dis 2019;19(1):810.

41. Defense Health Agency Solution Delivery Division. Fact sheet: electronic surveillance system for the early notification of community-based epidemics. US Department of Defense; 2015 [cited 2020 Nov 1][https://health. mil/Military-Health-Topics/Combat-Support/ Armed-Forces-Health-Surveillance-Branch/ Integrated-Biosurveillance/ESSENCE]

42. Lowry SZ, Ramaiah M, Patterson ES, Latkany P, Simmons D, Brick D, Gibbons MC. Electronic Health Record Design Considerations in
Responding to Incidences of Highly Infectious Diseases: Clinical Workflows and Exception Handling. Natl Inst Stand Technolgy US Dep Commer 2015; NISTIR 8095.

43. Borycki E, Cummings E, Dexheimer JW, Gong Y, Kennebeck S, Kushniruk A, et al. Patient-Centred Coordinated Care in Times of Emerging Diseases and Epidemics. Contribution of the IMIA Working Group on Patient Safety. Yearb Med Inform 2015;10(1):207.

44. Mandl KD. Ebola in the United States: EHRs as a public health tool at the point of care. JAMA 2014;312(23):2499-500.

45. Liang J, Zheng X, Chen Z, Dai S, Xu J, Ye $\mathrm{H}$, et al. The experience and challenges of healthcare-reform-driven medical consortia and Regional Health Information Technologies in China: A longitudinal study. Int J Med Inform 2019;131:103954.

46. Payne TH, Lovis C, Gutteridge C, Pagliari C, Natarajan S, Yong C, et al. Status of health information exchange: A comparison of six countries. J Glob Health 2019;9(2):020427.

47. Segar S, Segar S, Bouland D, Bouland D, Torriani F, Torriani F, et al. Flight of the COVID-19 patient: Experience with a Wuhan evacuee: A case report. J Med Case Rep 2020;14(1):1-6.

48. Grange ES, Neil EJ, Stoffel M, Singh AP, Tseng E, Resco-Summers K, et al. Responding to COVID-19: The UW Medicine Information Technology Services Experience. Appl Clin Inform 2020;11(2):265-75.

49. Lin C-T, Bookman K, Sieja A, Markley K, Altman RL, Sippel J, et al. Clinical Informatics Accelerates Health System Adaptation to the COVID-19 Pandemic: Examples from Colorado. J Am Med Inform Assoc 2020;27(12):1955-63.

50. Milenkovic A, Jankovic D, Rajkovic P. Extensions and adaptations of existing medical information system in order to reduce social contacts during COVID-19 pandemic. Int J Med Inform 2020;141:104224.

51. Yan A, Zou Y, Mirchandani DA. How hospitals in mainland China responded to the outbreak of COVID-19 using information technology-enabled services: An analysis of hospital news webpages. J Am Med Inform Assoc 2020;27(7):991-9.

52. Ye Q, Zhou J, Wu H. Using Information Technology to Manage the COVID-19 Pandemic: Development of a Technical Framework Based on Practical Experience in China. JMIR Med Inform 2020;8(6):e19515.

53. Sylvestre E, Thuny R-M, Cecilia-Joseph E, Gueye P, Chabartier C, Brouste Y, et al. Health Informatics Support for Outbreak Management: how to respond without an Electronic Health Record? J Am Med Inform Assoc. 2020;27(11):1828-9.

54. Kannampallil TG, Foraker RE, Lai AM, Woeltje KF, Payne PRO. When past is not a prologue: Adapting informatics practice during a pandemic. JAm Med Inform Assoc 2020;27(7):1142-6.

55. Hong Y-R, Lawrence J, Williams Jr D, Mainous III A. Population-Level Interest and Telehealth Capacity of US Hospitals in Response to COVID-19: Cross-Sectional Analysis of Google Search and National Hospital Survey Data. JMIR 
Public Health Surveill 2020;6(2):e18961.

56. Puro NA, Feyereisen S. Telehealth Availability in US Hospitals in the Face of the COVID-19 Pandemic. J Rural Health 2020;36(4):577-83.

57. Bhaskar S, Bradley S, Chattu VK, Adisesh A, Nurtazina A, Kyrykbayeva S, et al. Telemedicine Across the Globe-Position Paper From the COVID-19 Pandemic Health System Resilience PROGRAM (REPROGRAM) International Consortium (Part 1). Front Public Health 2020;8:556720.

58. Scott Kruse C, Karem P, Shifflett K, Vegi L, Ravi $\mathrm{K}$, Brooks M. Evaluating barriers to adopting telemedicine worldwide: A systematic review. J Telemed Telecare 2018;24(1):4-12.

59. Olson CA, Mcswain SD, Curfman AL, Chuo J. The current pediatric telehealth landscape. Pediatrics 2018;141(3):e20172334.

60. Hassan A, Mari Z, Gatto EM, Cardozo A, Youn J, Okubadejo N, et al. Global Survey on Telemedicine Utilization for Movement Disorders During the COVID-19 Pandemic. Mov Disord 2020;35(10):1701-1711.

61. Arshad Ali S, Bin Arif T, Maab H, Baloch M, Manazir S, Jawed F, et al. Global Interest in Telehealth During COVID-19 Pandemic: An Analysis of Google Trends ${ }^{\mathrm{TM}}$. Cureus 2020;12(9):e10487.

62. Smith AC, Thomas E, Snoswell CL, Haydon H, Mehrotra A, Clemensen J, et al. Telehealth for global emergencies: Implications for coronavirus disease 2019 (COVID-19). J Telemed Telecare 2020;26(5):309-13.

63. Mehrotra A, Ray K, Brockmeyer DM, Barnett ML, Bender JA. Rapidly Converting to "Virtual Practices": Outpatient Care in the Era of Covid-19. NEJM Catal 2020;1(2).

64. Meyer BC, Friedman LS, Payne K, Moore L, Cressler J, Holberg S, et al. Medical Undistancing Through Telemedicine: A Model Enabling Rapid Telemedicine Deployment in an Academic Health Center During the COVID-19 Pandemic. Telemed J E Health 2020; 27(6):625-34.

65. Mann DM, Chen J, Chunara R, Testa PA, Nov O. COVID-19 transforms health care through telemedicine: evidence from the field. J Am Med Inform Assoc 2020;27(7):1132-5.

66. van Dijk SDM, Bouman R, Folmer EH, den Held RC, Warringa JE, Marijnissen RM, et al. (Vi)-rushed Into Online Group Schema Therapy Based Day-Treatment for Older Adults by the COVID-19 Outbreak in the Netherlands. Am J Geriatr Psychiatry 2020;28(9):983-8.

67. Zhou X, Snoswell CL, Harding LE, Bambling M, Edirippulige S, Bai X, et al. The Role of Telehealth in Reducing the Mental Health Burden from COVID-19. Telemed J E Health. 2020;26(4):377-9.

68. Al-Shamsi HO, Alhazzani W, Alhuraiji A, Coomes EA, Chemaly RF, Almuhanna M, et al. A Practical Approach to the Management of Cancer Patients During the Novel Coronavirus Disease 2019 (COVID-19) Pandemic: An International Collaborative Group. Oncologist 2020;25(6):e936

69. Aziz A, Zork N, Aubey JJ, Baptiste CD, D'Alton ME, Emeruwa UN, et al. Telehealth for HighRisk Pregnancies in the Setting of the COVID-19 Pandemic. Am J Perinatol 2020;37(8):800.
70. Monaghesh E, Hajizadeh A. The role of telehealth during COVID-19 outbreak: A systematic review based on current evidence. BMC Public Health 2020;20(1):1193.

71. Cottrell MA, Russell TG. Telehealth for musculoskeletal physiotherapy. Musculoskelet Sci Pract 2020;48:102193.

72. Middleton A, Simpson KN, Bettger JP, Bowden MG. COVID-19 Pandemic and Beyond: Considerations and Costs of Telehealth Exercise Programs for Older Adults With Functional Impairments Living at Home-Lessons Learned From a Pilot Case Study. Phys Ther 2020;100(8):1278-88.

73. Wosik J, Fudim M, Cameron B, Gellad ZF, Cho A, Phinney D, et al. Telehealth transformation: COVID-19 and the rise of virtual care. J Am Med Inform Assoc 2020;27(6):957-62.

74. Hron JD, Parsons CR, Williams LA, Harper MB, Bourgeois FC. Rapid Implementation of an Inpatient Telehealth Program during the COVID-19 Pandemic. Appl Clin Inform 2020;11(3):452-9.

75. Vilendrer S, Patel B, Chadwick W, Hwa M, Asch $\mathrm{S}$, Pageler N, et al. Rapid Deployment of Inpatient Telemedicine In Response to COVID-19 Across Three Health Systems. J Am Med Inform Assoc 2020;27(7):1102-9.

76. Ong SY, Stump L, Zawalich M, Edwards L, Stanton $\mathrm{G}$, Matthews M, et al. Inpatient Telehealth Tools to Enhance Communication and Decrease Personal Protective Equipment Consumption during Disaster Situations: A Case Study during the COVID-19 Pandemic. Appl Clin Inform 2020;11(5):733-41.

77. Jones MS, Goley AL, Alexander BE, Keller SB, Caldwell MM, Buse JB. Inpatient Transition to Virtual Care During COVID-19 Pandemic. Diabetes Technol Ther 2020;22(6):444-8.

78. Wijesooriya NR, Mishra V, Brand PLP, Rubin BK. COVID-19 and telehealth, education, and research adaptations. Paediatr Respir Rev 2020;35:38-42.

79. Rogers A, Lynch K, Toth H, Weisgerber M. Patient and Family Centered (Tele)rounds: The Use of Video Conferencing to Maintain Family and Resident Involvement in Rounds. Acad Pediatr 2020;20(6):765-6.

80. Huffman LC, Feldman HM, Hubner LM. Fellows Front and Center: Tele-Training and Telehealth. Acad Pediatr 2020;20(6):764-5.

81. Coffey CS, MacDonald B V., Shahrvini B, Baxter SL, Lander L. Student Perspectives on Remote Medical Education in Clinical Core Clerkships During the COVID-19 Pandemic. Med Sci Educ 2020;1-8.

82. FDA. FDA Guidance on Conduct of Clinical Trials of Medical Products during COVID-19 Public Health Emergency: Guidance for Industry, Investigators, and Institutional Review Boards. Guidance Document; 2020 [cited 2020 Jan 11] [ https://www.fda.gov/regulatory-information/ search-fda-guidance-documents/fda-guidanceconduct-clinical-trials-medical-products-duringcovid-19-public-health-emergency]

83. Fisk M, Livingstone A, Pit SW. Telehealth in the context of COVID-19: Changing perspectives in Australia, the United Kingdom, and the United States. J Med Internet Res 2020;22(6):e19264.
84. Centers for Medicare and Medicaid Services. Fact sheet: Medicare telemedicine health care provider. 2020 [cited 2020 Nov 1][ https://www. cms.gov/newsroom/fact-sheets/medicare-telemedicine-health-care-provider-fact-sheet]

85. Centers for Medicare and Medicaid Services. President Trump Expands Telehealth Benefits for Medicare Beneficiaries During COVID-19 Outbreak; 2020 [cited 2020 Nov ][ https:// www.cms.gov/newsroom/press-releases/president-trump-expands-telehealth-benefits-medicare-beneficiaries-during-covid-19-outbreak]

86. Coronavirus Aid, Relief, and Economic Security (CARES) Act, Public Law 113 - 136; 134 Stat. 281. 2020 [cited 2020 Oct 11][ https:// www.congress.gov/116/bills/hr748/BILLS116hr748enr.pdf]

87. Heyworth L, Kirsh S, Zulman D, Ferguson JM, Kizer KW. Expanding Access through Virtual Care : The VA's Early Experience with Covid-19. NEJM Catal Innov Care Deliv 2020;1(4).

88. Department of Health and Human Services. Notification of enforcement discre- tion for telehealth remote communications during the COVID-19 nationwide public health emergency. [cited 2020 Apr 6] [https://www.hhs.gov/hipaa/for-professionals/special-topics/emergency-preparedness/ notification-enforcement-discretion-telehealth/ index.html]

89. Dorsey ER, Topol EJ. State of Telehealth. NEngl J Med 2016;375(2):154-61.

90. Katzow MW, Steinway C, Jan S. Telemedicine and health disparities during COVID-19. Pediatrics 2020;146(2):e20201586.

91. Nouri SS, Khoong EC, Lyles CR, Karliner LS Addressing Equity in Telemedicine for Chronic Disease Management During the Covid-19 Pandemic. NEJM Catal 2020;3(1):e20049.

92. Veinot TC, Ancker JS, Bakken S. Health informatics and health equity: Improving our reach and impact. J Am Med Inform Assoc 2019;26(8-9):689-95.

93. Shigekawa E, Fix M, Corbett G, Roby DH, Coffman J. The current state of telehealth evidence: a rapid review. Health Aff 2018;37(12):1975-82.

94. Baker MG, Wilson N, Anglemyer A. Successful Elimination of Covid-19 Transmission in New Zealand. N Engl J Med 2020;383(8):e56.

95. Wong JEL, Leo YS, Tan CC. COVID-19 in Singapore - Current Experience: Critical Global Issues That Require Attention and Action. JAMA 2020;323(13):1243-4.

96. Connor P. More than nine-in-ten people worldwide live in countries with travel restrictions amid COVID-19. Pew Research Center 2020 [cited 2020 Oct 1][https://www.pewresearch. org/fact-tank/2020/04/01/more-than-nine-in-tenpeople-worldwide-live-in-countries-with-travelrestrictions-amid-covid-19/]

97. Chen CM, Jyan HW, Chien SC, Jen HH, Hsu CY, Lee PC, et al. Containing COVID-19 among 627,386 persons in contact with the diamond princess cruise ship passengers who disembarked in Taiwan: Big data analytics. J Med Internet Res 2020;22(5):e19540.

98. Cheng HY, Jian SW, Liu DP, Ng TC, Huang WT, Lin HH. Contact Tracing Assessment of COVID-19 Transmission Dynamics in Taiwan 
and Risk at Different Exposure Periods before and after Symptom Onset. JAMA Intern Med 2020;180(9):1156-1163.

99. Jian SW, Cheng HY, Huang XT, Liu DP. Contact tracing with digital assistance in Taiwan's COVID-19 outbreak response. Int J Infect Dis 2020;101:348-52.

100. Huang YT, Tu YK, Lai PC. Estimation of the secondary attack rate of COVID-19 using proportional meta-analysis of nationwide contact tracing data in Taiwan. J Microbiol Immunol Infect 2020;54(1):89-92

101. Cohen IG, Gostin LO, Weitzner DJ. Digital Smartphone Tracking for COVID-19: Public Health and Civil Liberties in Tension. JAMA 2020;323(23):2371-2.

102. Dong E, Du H, Gardner L. An interactive webbased dashboard to track COVID-19 in real time. Lancet Infect Dis 2020;3099(20):19-20.

103. Ford D, Harvey J, McElligott J, King K, Simpson $\mathrm{K}$, Valenta S, et al. Leveraging Health System Telehealth and Informatics Infrastructure to Create a Continuum of Services for COVID-19 Screening, Testing, and Treatment. J Am Med Inform Assoc 2020; 27(12):1871-7.

104. Judson TJ, Odisho AY, Neinstein AB, Chao J, Williams A, Miller C, et al. Rapid design and implementation of an integrated patient self-triage and self-scheduling tool for COVID-19. J Am Med Inform Assoc 2020;27(6):860-6.

105. Judson TJ, Odisho AY, Young JJ, Bigazzi O, Steuer D, Gonzales R, et al. Implementation of a digital chatbot to screen health system employees during the COVID-19 pandemic. J Am Med Inform Assoc 2020;27(9):1450-5.

106. Annis T, Pleasants S, Hultman G, Lindemann E, Thompson JA, Billecke S, et al. Rapid implementation of a COVID-19 remote patient monitoring program. J Am Med Inform Assoc 2020;27(8):1326-1330.

107. Perlman A, Vodonos Zilberg A, Bak P, Dreyfuss $M$, Leventer-Roberts M, Vurembrand $Y$, et al. Digital and Remote Care among 71,619 Individuals Seeking COVID-19 Health Information and Services: A Retrospective Cohort Study. J Med Internet Res 2020;22(10):e23197.

108. Perez-Alba E, Nuzzolo-Shihadeh L, Espinosa-Mora JE, Camacho-Ortiz A. Use of self-administered surveys through QR code and same center telemedicine in a walk-in clinic in the era of COVID-19. J Am Med Inform Assoc 2020;27(6):985-6.

109. Turer RW, Jones I, Rosenbloom ST, Slovis C, Ward MJ. Electronic personal protective equipment: A strategy to protect emergency department providers in the age of COVID-19. J Am Med Inform Assoc 2020;27(6):967-71.

110. Sharfstein JM, Becker SJ, Mello MM. Diagnostic Testing for the Novel Coronavirus. JAMA 2020;323(15):1437-8.

111. Obeid JS, Davis M, Turner M, Meystre SM, Heider PM, Lenert LA. An AI approach to COVID-19 infection risk assessment in virtual visits: a case report. J Am Med Inform Assoc 2020;27(8):1321-5.

112. Liu Y, Wang Z, Ren J, Tian Y, Zhou M, Zhou $\mathrm{T}$, et al. A COVID-19 risk assessment decision support system for general practitioners: Design and development study. J Med Internet Res 2020;22(6):e19786.

113. Mao B, Liu Y, Chai YH, Jin XY, Lu HW, Yang JW, et al. Assessing risk factors for SARS$\mathrm{CoV}-2$ infection in patients presenting with symptoms in Shanghai, China: a multicentre, observational cohort study. Lancet Digit Health 2020;2(6): $323-30$

114. Albahri AS, Al-Obaidi JR, Zaidan AA, Albahri OS, Hamid RA, Zaidan BB, et al. Multi-biological Laboratory Examination Framework for the Prioritisation of Patients with COVID-19 Based on Integrated AHP and Group VIKOR Methods. Int J Inf Technol Decis Mak 2020;19(05):1247-1269.

115. Weemaes M, Martens S, Cuypers L, Van Elslande J, Hoet K, Welkenhuysen J, et al. Laboratory information system requirements to manage the COVID-19 pandemic: a report from the Belgian national reference testing center. J Am Med Inform Assoc 2020;27(8):1293-9.

116. Mei X, Lee HC, Diao K yue, Huang M, Lin B, Liu C, et al. Artificial intelligence-enabled rapid diagnosis of patients with COVID-19. Nat Med 2020;26:1224-1228.

117. Institute for Health Metrics and Evaluation. COVID-19 Projections [Internet]. [cited 2020 Oct 10]. Available from: http://covid19.healthdata.org/global?view=total-deaths $\&$ tab $=$ trend

118. Epstein RH, Dexter F. A Predictive Model for Patient Census and Ventilator Requirements at Individual Hospitals During the Coronavirus Disease 2019 (COVID-19) Pandemic: A Preliminary Technical Report. Cureus 2020;12(6):e8501.

119. Weissman GE, Crane-Droesch A, Chivers C, Luong TB, Hanish A, Levy MZ, et al. Locally Informed Simulation to Predict Hospital Capacity Needs During the COVID-19 Pandemic. Ann Intern Med 2020;173(1):21-8.

120. Roosa K, Lee Y, Luo R, Kirpich A, Rothenberg R, Hyman JM, et al. Real-time forecasts of the COVID-19 epidemic in China from February 5th to February 24th, 2020. Infect Dis Model 2020;5:256-63.

121. IHME COVID-19 health service utilization forecasting team, Murray CJ. Forecasting the impact of the first wave of the COVID-19 pandemic on hospital demand and deaths for the USA and European Economic Area countries. medRxiv; 2020 [http://dx.doi.org/10.1101/2020.04.21.200 74732]

122. McCall B. COVID-19 and artificial intelligence: protecting health-care workers and curbing the spread. Lancet Digit Health 2020;2(4):e166-7.

123. Debnath S, Barnaby DP, Coppa K, Makhnevich A, Kim EJ, Chatterjee S, et al. Machine learning to assist clinical decision-making during the COVID-19 pandemic. Bioelectron Med 2020;6(1):1-8.

124. Zhang K, Liu X, Shen J, Li Z, Sang Y, Wu X, et al. Clinically Applicable AI System for Accurate Diagnosis, Quantitative Measurements, and Prognosis of COVID-19 Pneumonia Using Computed Tomography. Cell. 2020;181(6):14231433.e11.

125. Li L, Qin L, Xu Z, Yin Y, Wang X, Kong B, et al. Using Artificial Intelligence to Detect COVID-19 and Community-acquired Pneumonia Based on
Pulmonary CT: Evaluation of the Diagnostic Accuracy. Radiology 2020;296(2):e65-71.

126. Murphy K, Smits H, Knoops AJG, Korst MBJM, Samson T, Scholten ET, et al. COVID-19 on Chest Radiographs: A Multireader Evaluation of an Artificial Intelligence System. Radiology 2020;296(3):e166-e172.

127. Hurt B, Kligerman S, Hsiao A. Deep Learning Localization of Pneumonia: 2019 Coronavirus (COVID-19) Outbreak. J Thorac Imaging 2020;35(3):W87-9.

128. Carlile M, Hurt B, Hsiao A, Hogarth M, Longhurst CA, Dameff C. Deployment of artificial intelligence for radiographic diagnosis of COVID 19 pneumonia in the emergency department. J Am Coll Emerg Physicians Open. 2020;1(6).

129. Goodman-Meza D, Rudas A, Chiang JN, Adamson PC, Ebinger J, Sun N, et al. A machine learning algorithm to increase COVID-19 inpatient diagnostic capacity. PLoS One 2020;15(9):e0239474.

130. Liang W, Yao J, Chen A, Lv Q, Zanin M, Liu $\mathrm{J}$, et al. Early triage of critically ill COVID-19 patients using deep learning. Nat Commun 2020;11(1):1-7.

131. Jacob J, Alexander D, Baillie JK, Berka R, Bertolli $\mathrm{O}$, Blackwood J, et al. Using imaging to combat a pandemic: rationale for developing the UK National COVID-19 Chest Imaging Database. Eur Respir J 2020;56(2):2001809.

132. Neves NMBC, Bitencourt FBCSN, Bitencourt AG V. Ethical dilemmas in COVID-19 times: how to decide who lives and who dies? Rev Assoc Med Bras 2020;66Suppl 2(Suppl 2):106-11.

133. Zamperetti N, Bellomo R, Ronco C. Bioethical aspects of end-of-life care. Eur J Anaesthesiol 2008;25:51-7.

134. Wynants L, Van Calster B, Collins GS, Riley RD, Heinze G, Schuit E, et al. Prediction models for diagnosis and prognosis of covid-19: Systematic review and critical appraisal. BMJ 2020;369:m1328.

135. Chen J, See KC. Artificial Intelligence for COVID-19: Rapid Review. J Med Internet Res 2020;22(10):e21476.

136. Bakker L, Aarts J, Uyl-de Groot C, Redekop W. Economic evaluations of big data analytics for clinical decision-making: a scoping review. J Am Med Inform Assoc 2020;27(9):1466-75.

137. Plasek JM, Tang C, Zhu Y, Huang Y, Bates DW. Following data as it crosses borders during the COVID-19 pandemic. J Am Med Inform Assoc 2020;27(7):1139-41.

138. Zeng ML, Hong Y, Clunis J, He S, Coladangelo LP. Implications of Knowledge Organization Systems for Health Information Exchange and Communication during the COVID-19 Pandemic. Data Inf Manag 2020;4(3):148-70.

139. Stang PE, Ryan PB, Racoosin JA, Overhage JM, Hartzema AG, Reich $C$, et al. Advancing the science for active surveillance: Rationale and design for the observational medical outcomes partnership. Ann Intern Med 2010;153(9):600-6.

140. Observational Health Data Sciences and Informatics. OMOP Common Data Model [https:// www.ohdsi.org]

141. Suchard MA, Schuemie MJ, Krumholz HM, 
You SC, Chen RJ, Pratt N, et al. Comprehensive comparative effectiveness and safety of first-line antihypertensive drug classes: a systematic, multinational, large-scale analysis. Lancet 2019;394(10211):1816-26.

142. Haimovich A, Ravindra NG, Stoytchev S, Young HP, PerryWilson F, van Dijk D, et al. Development and validation of the quick COVID-19 severity index (qCSI): a prognostic tool for early clinical decompensation. Ann Emerg Med 2020;76(4):442-53.

143. de Lusignan S, Jones N, Dorward J, Byford $\mathrm{R}$, Liyanage H, Briggs J, et al. Oxford Royal College of General Practitioners Clinical Informatics Digital Hub: Protocol to develop extended COVID-19 surveillance and trial platforms. JMIR Public Health Surveill 2020;6(3):e19773.

144. Neuraz A, Lerner I, Digan W, Paris N, Tsopra R, Rogier A, et al. Natural language processing for rapid response to emergent diseases: Case study of calcium channel blockers and hypertension in the COVID-19 pandemic. J Med Internet Res 2020;22(8): e20773.

145. Galvin CJ, Fernandez-Luque L, Li Y-C (Jack). Accelerating the global response against the exponentially growing COVID-19 outbreak through decent data sharing. Diagn Microbiol Infect Dis 2020;115070.

146. Burn E, You SC, Sena AG, Kostka K, Abedtash $\mathrm{H}$, Abrahão MTF, et al. Deep phenotyping of 34,128 adult patients hospitalised with COVID-19 in an international network study. Nat Commun 2020;11(1):5009.

147. Raisaro JL, Marino F, Troncoso-Pastoriza J, Beau-Lejdstrom R, Bellazzi R, Murphy R, et al. SCOR: A secure international informatics infrastructure to investigate COVID-19. J Am Med Inform Assoc 2020;27(11):1721-6.

148. Haendel MA, Chute CG, Gersing K. The National COVID Cohort Collaborative (N3C): Rationale, Design, Infrastructure, and Deployment. J Am Med Inform Assoc 2021;28(3):427-3.

149. Brat GA, Weber GM, Gehlenborg N, Avillach P, Palmer NP, Chiovato L, et al. International electronic health record-derived COVID-19 clinical course profiles: the 4CE consortium. npj Digit Med 2020;3(109)

150. LOINC. Guidance for mapping to SARS-CoV-2 LOINC terms. [cited 2020 Oct 10][https://loinc. org/sars-coronavirus-2/]

151. Dong X, Li J, Soysal E, Bian J, DuVall SL, Hanchrow E, et al. COVID-19 TestNorm: A tool to normalize COVID-19 testing names to LOINC codes. J Am Med Inform Assoc 2020;27(9):1437-42.

152. Centers for Disease Control and Prevention. Public Health Information Network Vocabulary Access and Distribution System (PHIN VADS) [cited 2020 Oct 10][https://phinvads.cdc.gov/ vads/SearchVocab.action]

153. Garcia M, Lipskiy N, Tyson J, Watkins R, Esser ES, Kinley T. Centers for Disease Control and Prevention 2019 novel coronavirus disease (COVID-19) information management: addressing national health-care and public health needs for standardized data definitions and codified vocabulary for data exchange. J Am Med Inform Assoc 2020;27(9):1476-87.
154. Cosgriff C V., Ebner DK, Celi LA. Data sharing in the era of COVID-19. Lancet Digit Health 2020;2(5):e224.

155. Bruthans J. The state of national electronic prescription systems in the EU in 2018 with special consideration given to interoperability issues. Int J Med Inform 2020;141:104205.

156. O'Reilly-Shah VN, Gentry KR, Van Cleve W, Kendale SM, Jabaley CS, Long DR. The COVID-19 Pandemic Highlights Shortcomings in US Health Care Informatics Infrastructure: A Call to Action. Anesth Analg 2020;131(2):340-4.

157. Ienca M, Vayena E. On the responsible use of digital data to tackle the COVID-19 pandemic. Nat Med 2020;26(4):463-4.

158. Lenert L, McSwain BY. Balancing health privacy, health information exchange, and research in the context of the COVID-19 pandemic. J Am Med Inform Assoc 2020;27(6):963-6.

159. Kandel N, Chungong S, Omaar A, Xing J. Health security capacities in the context of COVID-19 outbreak: an analysis of International Health Regulations annual report data from 182 countries. Lancet 2020;395(10229):1047-53.

160. Holmgren AJ, Apathy NC, Adler-Milstein J. Barriers to hospital electronic public health reporting and implications for the COVID-19 pandemic. J Am Med Inform Assoc 2020;27(8):1306-1309.

161. Stenner SP, Rice W, Nelson SD. A Viewpoint on the Information Sharing Paradox. Appl Clin Inform 2020;11(3):460-3.

162. Foraker RE, Lai AM, Kannampallil TG, Woeltje KF, Trolard AM, Payne PRO. Transmission Dynamics: Data Sharing in the COVID-19 Era. Learn Health Syst 2021;5(1):e10235.

163. Sim I, Stebbins M, Bierer BE, Butte AJ, Drazen J, Dzau V, et al. Time for NIH to lead on data sharing. Science 2020;367(6484):1308-9.

164. Dong E, Du H, Gardner L. An interactive webbased dashboard to track COVID-19 in real time. Lancet Infect Dis 2020;20(5):533-4.

165. World Health Organization. World Health Organization. Novel coronavirus (COVID-19) situation (public dashboard) [cited 2020 Oct 4] [https://covid19.who.int]

166. Centers for Disease Control and Prevention. CDC COVID Data Tracker [Internet]. Coronavirus Disease (2019)[https://covid. cdc.gov/covid-data-tracker/?CDC_AA_ refVal=https $\% 3 \mathrm{~A} \% 2 \mathrm{~F} \% 2 \mathrm{Fwww} . \mathrm{cdc}$.gov$\% 2 \mathrm{Fcoronavirus} \% 2 \mathrm{~F} 2019$-ncov $\% 2 \mathrm{Fcases}$-updates $\% 2$ Fcases-in-us.html\#cases_casesinlast7 days]

167. Xu B, Gutierrez B, Mekaru S, Sewalk K, Goodwin L, Loskill A, et al. Epidemiological data from the COVID-19 outbreak, real-time case information. Sci Data. 2020;7(1):1-6.

168. Kamel Boulos MN, Geraghty EM. Geographical tracking and mapping of coronavirus disease COVID-19/severe acute respiratory syndrome coronavirus 2 (SARS-CoV-2) epidemic and associated events around the world: How 21st century GIS technologies are supporting the global fight against outbr. Int J Health Geogr 2020;19(1):1-12.

169. Wissel BD, Van Camp PJ, Kouril M, Weis C, Glauser TA, White PS, et al. An interactive online dashboard for tracking COVID-19 in U.S. counties, cities, and states in real time. J Am Med Inform Assoc 2020;27(7):1121-1125.

170. Arneson D, Elliott M, Mosenia A, Oskotsky B, Vashisht R, Zack T, et al. CovidCounties is an interactive real-time tracker of the COVID-19 pandemic at the level of US counties. Sci Data 2020;7(1):1-10.

171. James P, Das R, Jalosinska A, Smith L. Smart cities and a data-driven response to COVID-19. Dialogues Hum Geogr 2020;10(2).

172. Thorlund K, Dron L, Park J, Hsu G, Forrest JI, Mills EJ. A real-time dashboard of clinical trials for COVID-19. Lancet Digit Health 2020;2(6):e286-7.

173. Dixit RA, Hurst S, Adams KT, Boxley C, Lysen-Hendershot K, Bennett SS, et al. Rapid development of visualization dashboards to enhance situation awareness of COVID-19 telehealth initiatives at a multihospital healthcare system. J Am Med Inform Assoc 2020;27(9): 1456-61.

174. World Health Organization. Pandemic Influenza Preparedness and Response: A WHO Guidance Document; 2009. [cited 2020 Oct 1][https://apps. who.int/iris/handle/10665/44123]

175. World Health Organization. Implementation and management of contact tracing for Ebola virus disease 2015. [cited 2020 Oct 1][https:// www.who.int/csr/resources/publications/ebola/ contact-tracing/en/]

176. Kretzschmar ME, Rozhnova G, Bootsma MCJ, van Boven $\mathrm{M}$, van de Wijgert JHHM, Bonten MJM. Impact of delays on effectiveness of contact tracing strategies for COVID-19: a modelling study. Lancet Public Health 2020;5(8):e452-9.

177. Ferretti L, Wymant C, Kendall M, Zhao L, Nurtay A, Abeler-Dörner L, et al. Quantifying SARSCoV-2 transmission suggests epidemic control with digital contact tracing. Science 2020 May 8;368(6491):eabb6936.

178. Abuhammad S, Khabour OF, Alzoubi KH. Covid-19 contact-tracing technology: Acceptability and ethical issues of use. Patient Prefer Adherence 2020;14:1639.

179. Zastrow M. South Korea is reporting intimate details of COVID-19 cases: has it helped? Nature 2020 [https://www.nature.com/articles/d41586020-00740-y]

180. Goggin G. COVID-19 apps in Singapore and Australia: reimagining healthy nations with digital technology. Media Int Aust 2020;177(1):61-75.

181. Sin Y. Parliament: $\$ 13.8$ million spent on SafeEntry, TraceTogether digital contact tracing tools [https://www.straitstimes.com/singapore/ politics/parliament-138-million-spent-on-safeentry-tracetogether-digital-contact-tracing]

182. Fahey RA, Hino A. COVID-19, digital privacy, and the social limits on data-focused public health responses. Int J Inf Manage 2020;55:102181.

183. Huang Z, Guo H, Lee YM, Ho EC, Ang H, Chow A. Performance of Digital Contact Tracing Tools for COVID-19 Response in Singapore: Cross-Sectional Study. JMIR mHealth uHealt. 2020;8(10):e23148.

184. Braithwaite I, Callender T, Bullock M, Aldridge RW. Automated and partly automated contact tracing: a systematic review to inform 
the control of COVID-19. Lancet Digit Health 2020;2(11):e607-21.

185. Anglemyer A, Moore TH, Parker L, Chambers T, Grady A, Chiu K, et al. Digital contact tracing technologies in epidemics: a rapid review. Cochrane Database Syst Rev 2020;8:CD013699.

186. World Health Organization. Tracking COVID-19: Contact Tracing in the Digital Age [cited 2020 Jan 10][https://www.who.int/news-room/feature-stories/detail/tracking-covid-19-contacttracing-in-the-digital-age]

187. Ho HJ, Zhang ZX, Huang Z, Aung AH, Lim WY, Chow A. Use of a real-time locating system for contact tracing of health care workers during the COVID-19 pandemic at an infectious disease center in singapore: Validation study. J Med Internet Res 2020;22(5):e19437.

188. Venkataraman N, Hoong PB, Chuin S. Innovative Use of Health Informatics to Augment Contact Tracing during the COVID19 Pandemic in an Acute Hospital. J Am Med Inform Assoc 2020; 27(12):1964-7.

189. World Health Organization. International Classification of Diseases for Mortality and Morbidity Statistics. Eleventh Revision. 2019; [cited 2020 Oct 11][https://www.who.int/classifications/ classification-of-diseases]

190. Nations U. World Drug Report 2020. United Nations Off Drugs Crime. 2020 [https://wdr.unodc. org/wdr2020/field/WDR20_BOOKLET_1.pdf]

191. Weiner SG, Price CN, Atalay AJ, Harry EM, Pabo EA, Patel R, et al. A Health System-Wide Initiative to Decrease Opioid-Related Morbidity and Mortality. Jt Comm J Qual Patient Saf 2019:45(1):3-13.

192. Muniga ET, Walroth TA, Washburn NC. The Impact of Changes to an Electronic Admission Order Set on Prescribing and Clinical Outcomes in the Intensive Care Unit. Appl Clin Inform 2020;11(1):182-9.

193. Nemati S, Holder A, Razmi F, Stanley MD, Clifford GD, Buchman TG. An Interpretable Machine Learning Model for Accurate Prediction of Sepsis in the ICU. Crit Care Med 2018;46(4):547.

194. Hayden GE, Tuuri RE, Scott R, Losek JD, Blackshaw AM, Schoenling AJ, et al. Triage sepsis alert and sepsis protocol lower times to fluids and antibiotics in the ED. Am J Emerg
Med 2016;34(1):1-9.

195. Downing NL, Rolnick J, Poole SF, Hall E, Wessels AJ, Heidenreich P, et al. Electronic health record-based clinical decision support alert for severe sepsis: A randomised evaluation. BMJ Qual Saf 2019;28(9):762-8.

196. Baghdadi JD, Brook RH, Uslan DZ, Needleman J, Bell DS, Cunningham WE, et al. Association of a Care Bundle for Early Sepsis Management with Mortality among Patients with Hospital-Onset or Community-Onset Sepsis. JAMA Intern Med 2020;180(5):707-16.

197. Page AJ, Gani F, Crowley KT, Lee KHK, Grant MC, Zavadsky TL, et al. Patient outcomes and provider perceptions following implementation of a standardized perioperative care pathway for open liver resection. Br J Surg 2016;103(5):564-71.

198. Hu QL, Liu JY, Hobson DB, Cohen ME, Hall BL, Wick EC, et al. Best Practices in Data Use for Achieving Successful Implementation of Enhanced Recovery Pathway. J Am Coll Surg 2019;229(6):626-32.

199. Wick EC, Galante DJ, Hobson DB, Benson AR, Lee KHK, Berenholtz SM, et al. Organizational Culture Changes Result in Improvement in Patient-Centered Outcomes: Implementation of an Integrated Recovery Pathway for Surgical Patients. J Am Coll Surg 2015;221(3):669-77.

200. Sutcliffe KM, Paine L, Pronovost PJ. Re-examining high reliability: Actively organising for safety. BMJ Qual Saf 2017;26(3):248-51.

201. World Health Organization. Critical preparedness, readiness and response actions for COVID-19: Interim Guidance. 4 Nov 2020. [cited 2020 Oct 11][https://www.who.int/emergencies/ diseases/novel-coronavirus-2019/technical-guidance-publications?publicationtypes $=01 \mathrm{bc} 799 \mathrm{c}-\mathrm{b}$ 461-4a52-8c7d-294c84cd7b2d]

202. Naz A, Shahid F, Butt TT, Awan FM, Ali A, Malik A. Designing Multi-Epitope Vaccines to Combat Emerging Coronavirus Disease 2019 (COVID-19) by Employing Immuno-Informatics Approach. Front Immunol 2020;11:1663.

203. Kwarteng A, Asiedu E, Sakyi SA, Asiedu SO Targeting the SARS-CoV2 nucleocapsid protein for potential therapeutics using immuno-informatics and structure-based drug discovery techniques. Biomed Pharmacother 2020;132:110914.
204. Zhou Y, Hou Y, Shen J, Huang Y, Martin W, Cheng F. Network-based drug repurposing for novel coronavirus 2019-nCoV/SARS-CoV-2. Cell Discov 2020;6(1):1-18.

205. Subbian V, Solomonides A, Clarkson M, Rahimzadeh VN, Petersen C, Schreiber R, et al. Ethics and informatics in the age of COVID-19: challenges and recommendations for public health organization and public policy. JAm Med Inform Assoc 2021; 28(1):184-9.

206. Bollyky TJ, Gostin LO, Hamburg MA. The Equitable Distribution of COVID-19 Therapeutics and Vaccines. JAMA 2020;323(24):2462-3.

207. Liu J, Wang Z, Huang S, Ren. A. An Overview of Healthcare Information Technologies Used to Combat the COVID-19 Pandemic in China. International Journal of Digital Health. 2021;1(1):14;1-9."

208. He J, Baxter SL, Xu J, Xu J, Zhou X, Zhang $\mathrm{K}$. The practical implementation of artificial intelligence technologies in medicine. Nat Med 2019;25(1):30-6.

209. Lee TC, Shah NU, Haack A, Baxter SL. Clinical Implementation of Predictive Models Embedded within Electronic Health Record Systems: A Systematic Review. Informatics (MDPI) 2020;7(3):25.

210. Rahimi B, Nadri H, Afshar HL, Timpka T. A systematic review of the technology acceptance model in health informatics. Appl Clin Inform 2018;9(3):604.

211. Madhavan S, Bastarache L, Brown JS, Butte A, Dorr D, Embi PJ, et al. Use of Electronic Health Records to Support a Public Health Response to the COVID-19 Pandemic in the United States: A Perspective from Fifteen Academic Medical Centers. J Am Med Inform Assoc 2020; 28(2):393-401.

\section{Correspondence to:}

Christopher A Longhurst, MD

University of California, San Diego

9560 Towne Center Drive Ste 100

La Jolla, CA 92121

USA

E-mail: clonghurst@health.ucsd.edu 